\title{
Data Visualization and Dimensionality Reduction Using Kernel Maps With a Reference Point
}

\author{
Johan A. K. Suykens, Senior Member, IEEE
}

\begin{abstract}
In this paper, a new kernel-based method for data visualization and dimensionality reduction is proposed. A reference point is considered corresponding to additional constraints taken in the problem formulation. In contrast with the class of kernel eigenmap methods, the solution (coordinates in the low-dimensional space) is characterized by a linear system instead of an eigenvalue problem. The kernel maps with a reference point are generated from a least squares support vector machine (LS-SVM) core part that is extended with an additional regularization term for preserving local mutual distances together with reference point constraints. The kernel maps possess primal and dual model representations and provide out-of-sample extensions, e.g., for validation-based tuning. The method is illustrated on toy problems and real-life data sets.
\end{abstract}

Index Terms-Constrained optimization, data visualization, dimensionality reduction, feature map, kernel methods, least squares support vector machines (LS-SVMs), positive-definite kernel, validation.

\section{INTRODUCTION}

$\mathbf{T}$ RADITIONALLY, techniques such as principal component analysis (PCA) and self-organizing maps (SOMs) have been frequently applied for dimensionality reduction and data visualization [13], [15]. In recent years, the search for new approaches and solutions to this problem has become an active area of research and a variety of new techniques have been proposed such as isomap, locally linear embedding (LLE), Hessian LLE, diffusion maps, Laplacian eigenmaps, and others [2], [6], [9], [14], [27], [31]. Currently, many of these techniques have been characterized under the umbrella of kernel eigenmap methods and manifold learning [3], [7]. Nevertheless, the selection of components and tuning parameters is often difficult to assess. For several existing approaches, the characterization of out-of-sample extensions is unclear, though approximate versions have been proposed [4]. Furthermore, the issue of model selection of tuning parameters is to a large extent still an open problem [3].

Manuscript received February 2, 2007; revised August 22, 2007 and February 8, 2008; accepted March 13, 2008. First published July 9, 2008; current version published September 4, 2008. This work was supported by the Research Council K.U. Leuven under GOA AMBioRICS, CoE EF/05/006, OT/03/12, $\mathrm{Ph}$.D./postdoctoral, and fellow grants; by the Flemish Government under FWO Ph.D./postdoctoral grants and FWO projects G.0499.04, G.0211.05, G.0226.06, and G.0302.07; by the research communities ICCoS, ANMMM, and MLDM; by AWI under BIL/05/43; by IWT under Ph.D. Grants; and by the Belgian Federal Science Policy Office under IUAP P5/22 and IUAP DYSCO.

The author is with the Katholieke Universiteit Leuven, ESAT-SCD/SISTA, B-3001 Leuven (Heverlee), Belgium (e-mail: johan.suykens@esat.kuleuven. be).

Color versions of one or more of the figures in this paper are available online at http://ieeexplore.ieee.org.

Digital Object Identifier 10.1109/TNN.2008.2000807
In this paper, we want to take a fresh look at the problem of data visualization with dimensionality reduction. A main objective here is to design a technique for which the solution follows from solving a linear system instead of an eigenvalue problem. At the same time the method should be able to perform nonlinear dimensionality reduction with preserving local mutual distances and allow for out-of-sample extensions that can be made in an exact way. The tuning parameters should be set in view of obtaining a good generalization ability of the underlying model on new data.

Kernel-based learning methods have proven to be successful in many applications in different areas, especially, on problems with high-dimensional input spaces. Different methodologies and mathematical frameworks have emerged that are making use of kernels, including support vector machine (SVM) methodologies, function estimation in reproducing kernel Hilbert spaces (RKHS) and Bayesian learning viewpoints with Gaussian processes [10], [20], [21], [26], [28], [30]. In this paper, we propose a new kernel-based method in the framework of least squares support vector machines (LS-SVMs). In this context, kernel-based models have been studied for regression, classification, PCA, spectral clustering, canonical correlation analysis, recurrent networks, optimal control, and others [1], [18], [23]-[26]. The formulations are in terms of constrained optimization problems with use of a feature map in the primal problem and a related positive-definite kernel in the dual (which is the problem in the Lagrange multipliers that are related to the constraints). This is similar to standard SVM formulations [28] but by making use of an $L_{2}$ loss function and equality constraints instead of inequality constraints. Some advantages of this setting are the systematic and straightforward way of deriving optimal model representations and constraints handling.

The kernel map approach that we propose in this paper makes use of a reference point. Such a reference point is expressed in terms of constraints that are added to the formulation. We show how this mechanism converts the eigenvalue problem into a linear system. As stated in [22], complexity theory results for linear systems are easier to obtain than for eigenvalue problems. Also, the development of fast cross-validation techniques and online learning methods for the design of adaptive systems becomes easier [5] and both direct and iterative solvers can be used [12]. The solution to the linear system delivers the coordinates in the low-dimensional space. The support values follow from a second linear system. The optimal model representation and solution are generated in a straightforward way from an LS-SVM core part that maps the input data to the coordinates in the low-dimensional space. An additional regularization term that preserves local mutual distances is incorporated. This term 
is a modified version of the one that is considered in LLE [16]. While the reference point enables to achieve a linear system solution, the point has to be sacrificed and omitted in the final data visualization. Its role is related to choosing a geometric perspective on the object for the data visualization and can be fixed beforehand.

The problem statement as a constrained optimization problem admits primal and dual model representations, respectively, in terms of feature maps and the associated positive-definite kernel functions. An important consequence is that the models can immediately be extended for the evaluation at new data points with out-of-sample extensions. Out-of-sample extensions can also be obtained by considering regularized least squares approaches with function estimation in an RKHS [3], [29] when the RKHS is defined everywhere in the input space. However, in this paper, we optimize not only over an unknown function, but jointly over the unknown coordinates in the low-dimensional space, the primal weights of the unknown model, and the error variables. The optimization setting with primal and dual problems enables to obtain the optimal model representations and the final solution at once from the conditions for optimality.

Despite the importance of studies in learning theory with bounds on the generalization error, mainly for classification and regression problems [8], [28], practitioners often still rely on validation-based methods, due to the lack of sharp bounds in general. As stated in the conclusions of [3] the issue of model selection in problems of manifold regularization is currently not well understood. In this paper, we investigate two model selection criteria with use of a validation set (and randomized validation set) and cross validation. Both criteria are normalized in the sense that they are invariant with respect to scaling of the underlying model by a constant. A normalized training objective was also proposed for problems of supervised graph inference in [29]. We show results for a number of examples with toy problems and real-life data sets with 2-D and 3-D visualizations of the data sets.

This paper is organized as follows. In Section II, we discuss the context and outline of this paper. In Section III, we consider kernel maps that lead to an eigenvalue problem, generated from an LS-SVM core model. In Section IV, we discuss the introduction of a reference point leading to a linear system solution. First, this is explained for projection to a 2-D space and next for the general case. In Section V, aspects of model selection are discussed. In Section VI, examples are given on toy problems and real-life data sets. Comparisons with other methods are made. To enhance the readability of this paper, all proofs are given in the Appendix. ${ }^{1}$

\section{ConteXt And OutLine}

Consider a given input data set $\mathcal{D}=\left\{x_{i}\right\}_{i=1}^{N}$ of $N$ data points $x_{i} \in \mathbb{R}^{p}$. We aim at realizing a dimensionality reduction $x \mapsto z$ to a low-dimensional space by $g(\cdot): \mathbb{R}^{p} \rightarrow \mathbb{R}^{d}$ with $d=2$ or $d=3$ for data visualization of the corresponding points $\left\{z_{i}\right\}_{i=1}^{N}$ with $z_{i} \in \mathbb{R}^{d}$. Let us define the vector $z=\left[z_{1} ; z_{2} ; \ldots ; z_{N}\right]=$

\footnotetext{
${ }^{1} \mathrm{~A}$ Matlab demo file that shows the application of the kernel map reference point method to the Swiss roll problem can be downloaded from http://www. esat.kuleuven.be/sista/lssvmlab/KMref/demoswissKMref.m
}

$\left[z_{1}^{T} z_{2}^{T} \ldots z_{N}^{T}\right]^{T} \in \mathbb{R}^{d N}$ and the following mechanism to select the $i$ th data point from the vector $z$ for the different components:

$$
\begin{aligned}
z_{i, 1} & =c_{i, 1}^{T} z \\
z_{i, 2} & =c_{i, 2}^{T} z \\
\vdots & \\
z_{i, d} & =c_{i, d}^{T} z
\end{aligned}
$$

where $z_{i, l}$ denotes the $l$ th component $(l=1, \ldots, d)$ of the $i$ th data point vector $z_{i}(i=1, \ldots, N)$ with

$$
\begin{gathered}
c_{1,1}=\left[1 ; 0_{d-1} ; 0_{d} ; \ldots ; 0_{d}\right], \ldots, c_{1, d}=\left[0_{d-1} ; 1 ; 0_{d} ; \ldots ; 0_{d}\right] \\
\ldots \\
c_{N, 1}=\left[0_{d} ; 0_{d} ; \ldots ; 1 ; 0_{d-1}\right], \ldots, c_{N, d}=\left[0_{d} ; 0_{d} ; \ldots ; 0_{d-1} ; 1\right] .
\end{gathered}
$$

A meaningful objective for dimensionality reduction is to find the points $z_{i}$ as the solution to

$$
\min _{z_{i} \in \mathbb{R}^{d}} J=-\frac{\gamma}{2} \sum_{i=1}^{N}\left\|z_{i}\right\|_{2}^{2}+\frac{1}{2} \sum_{i=1}^{N}\left\|z_{i}-\sum_{j=1}^{N} s_{i j} z_{j}\right\|_{2}^{2}
$$

where the first term is a regularization term to avoid the trivial solution with $\gamma>0$. The second term minimizes the objective $\sum_{i=1}^{N}\left\|z_{i}-\tilde{z}_{i}\right\|_{2}^{2}$ with

$$
\tilde{z}_{i}=\sum_{j=1}^{N} s_{i j} z_{j}
$$

based on similarities $s_{i j}$ defined on pairs of input data $x_{i}, x_{j}$. A typical choice of the matrix $S$ are the $i j$ th entries $s_{i j}=$ $\exp \left(-\left\|x_{i}-x_{j}\right\|_{2}^{2} / \sigma^{2}\right)$. Written in terms of the unknown vector $z$, the objective (3) becomes

$$
\min _{z \in \mathbb{R}^{d N}} J=-\frac{\gamma}{2} z^{T} z+\frac{1}{2}(z-P z)^{T}(z-P z)
$$

where

$$
P=\left[\begin{array}{cccc}
s_{11} I_{d} & s_{12} I_{d} & \ldots & s_{1 N} I_{d} \\
s_{21} I_{d} & s_{22} I_{d} & \ldots & s_{2 N} I_{d} \\
\vdots & & & \vdots \\
s_{N 1} I_{d} & s_{N 2} I_{d} & \ldots & s_{N N} I d
\end{array}\right]
$$

Setting $\partial J / \partial z=0$ yields the eigenvalue problem

$$
R z=\gamma z
$$

with $R=(I-P)^{T}(I-P)$ from which one has to select one of the candidate eigenvector solutions.

This problem (3)-(5) has the following connections to the methods of LLE and Laplacian eigenmaps. 
- $L L E$ [16]: The objective $\sum_{i=1}^{N}\left\|z_{i}-\sum_{j=1}^{N} s_{i j} z_{j}\right\|_{2}^{2}$ is related to LLE as follows. In LLE, one establishes a connection between the cost function

$$
\mathcal{E}_{\text {in }}=\sum_{i=1}^{N}\left\|x_{i}-\sum_{j=1}^{N} w_{i j} x_{j}\right\|_{2}^{2}
$$

and the cost function

$$
\mathcal{E}_{\text {out }}=\sum_{i=1}^{N}\left\|z_{i}-\sum_{j=1}^{N} w_{i j} z_{j}\right\|_{2}^{2}
$$

through minimization of the weight matrix $W$ with $i j$ th entries $w_{i j}$, which are in common between both cost functions. One takes $w_{i j}=0$ if $x_{j}$ is not among the $k$-nearest neighbors of $x_{i}$ and $\sum_{j} w_{i j}=1$ for all $i$. Furthermore, the output coordinates are centered and have a unit covariance matrix to prevent degenerate solutions [17]. A difference between LLE and (3)-(5) in this paper is that we consider the weights $s_{i j}$ to be functions of the differences $x_{i}-x_{j}$ such that $s(\cdot)$ can be evaluated on any pair of data points. In LLE, these weights are taken to be fixed and determined at the training level.

- Laplacian eigenmaps [2]: For the method of Laplacian eigenmaps, a connection in an approximate sense with an iterated Laplacian has been derived in [2]. It was noticed that in relation to $\mathcal{E}_{\text {in }}$ of LLE, one has

$$
\mathcal{A} f \approx \frac{1}{2}\left(\Delta_{\mathcal{M}}\right)^{2} f
$$

where $\Delta_{\mathcal{M}}$ denotes the Laplace-Beltrami operator on a differentiable function $f$ on a manifold $\mathcal{M}$ and $\mathcal{A}$ is an operator related to the matrix $A=(I-W)^{T}(I-W)$.

In this paper, we are interested now in the use of kernel maps to project the original input data to the estimated coordinates in the lower dimensional space. This will be approached in two steps.

1) We will consider the use of an underlying model that gets the given data $x_{i}$ as input and outputs the estimated coordinates $\hat{z}_{i}$ for data visualization (instead of $z_{i}$ ). The core part is constituted by an LS-SVM regression that takes the criterion (3) as an additional regularization term in a regularized least squares objective. The solution is characterized by an eigenvalue problem and is discussed in Section III.

2) The coordinates $z_{i}$ for one single data point (e.g., for the first data point $z_{1}$ ) are fixed beforehand. This is incorporated by additional constraints in the problem formulation. As a result the solution is characterized by a linear system instead of an eigenvalue problem, as will be explained in Section IV.

The obtained underlying models with primal and dual model representations allow to make out-of-sample extensions. In this way, the coordinates for data visualization $\hat{z}_{i}$ can be obtained for training, validation, and test data. Model selection for the tuning parameters will be studied then in Section V for making use of validation sets and cross validation.

\section{Kernel Maps And Eigenvalue Problem}

We can realize the nonlinear mapping $g$ through an LS-SVM regression. This is combined then with the criterion (5) that is taken as an additional regularization term. This results in the following primal problem:

$$
\begin{aligned}
& \min _{z, w_{j}, e_{i, j}} \quad J_{2}=-\frac{\gamma}{2} z^{T} z+\frac{1}{2}(z-P z)^{T}(z-P z) \\
&+\frac{\nu}{2} \sum_{j=1}^{d} w_{j}^{T} w_{j}+\frac{\eta}{2} \sum_{i=1}^{N} \sum_{j=1}^{d} e_{i, j}^{2} \\
& \text { such that } \quad c_{i, j}^{T} z=w_{j}^{T} \varphi_{j}\left(x_{i}\right)+e_{i, j} \quad \forall i=1, \ldots, N, \\
& j=1, \ldots, d
\end{aligned}
$$

with $w_{j} \in \mathbb{R}^{n_{h_{j}}}$ and error variables $e_{i, j}$. The regularization constants $\nu$ and $\eta$ are assumed to be positive. Different feature maps $\varphi_{j}(\cdot): \mathbb{R}^{p} \rightarrow \mathbb{R}^{n_{h_{j}}}$ are used in order to realize mappings from the given input space to the different components in the lower dimensional space, where $n_{h_{j}}$ denote the dimensions of the feature maps. A positive-definite kernel function $K(\cdot, \cdot): \mathbb{R}^{p} \times \mathbb{R}^{p} \rightarrow \mathbb{R}$ is related to a feature map $\varphi(\cdot): \mathbb{R}^{p} \rightarrow$ $\mathbb{R}^{n_{h}}$ through $K(x, z)=\varphi(x)^{T} \varphi(z)$ (which is often called the kernel trick). For the commonly used radial basis function kernel $K(x, z)=\exp \left(-\|x-z\|_{2}^{2} / \sigma^{2}\right)$ the feature map is infinite dimensional $\left(n_{h} \rightarrow \infty\right)$.

The constrained optimization problem (10) is the primal problem with unknown coordinates $z$ of the data in the lower dimensional space, the error variables $e_{i, j}$, and the vector $w_{j}$ for the primal representation of the model

$$
\hat{z}_{*, j}=w_{j}^{T} \varphi_{j}\left(x^{*}\right)
$$

evaluated at any point $x^{*} \in \mathbb{R}^{p}$ in the input space (and hence also allows for out-of-sample extensions). It yields the corresponding predicted coordinates $\hat{z}_{*, j}$. Neural network interpretations with a hidden layer and hidden units can be given both to this primal representation of the model (in terms of the feature map) and to the dual representation of the model (in terms of the kernel function) [26].

The objective function $J$ from (5) is acting in (10) as an additional regularization term to the LS-SVM core part. In a different context, an additional Laplacian-based regularization term has also been considered in [3] for Laplacian regularized least squares methods with function estimation in an RKHS. Also, in problems of supervised graph inference [29], this has been proposed. Combined objectives with use of an LS-SVM core part have also been studied in a different area of finding approximate solutions to optimal control problems in [25].

To show the difference between the solutions to (5) and (10), we consider the zero bias term case. To fix the ideas, let us con- 
sider the case $d=2$ where the given input data are to be projected and visualized in a 2-D space

\section{(Problem P1)}

$$
\begin{aligned}
& \min _{z, w_{1}, w_{2}, e_{i, 1}, e_{i, 2}} J_{3}=-\frac{\gamma}{2} z^{T} z+\frac{1}{2}(z-P z)^{T}(z-P z) \\
& +\frac{\nu}{2}\left(w_{1}^{T} w_{1}+w_{2}^{T} w_{2}\right)+\frac{\eta}{2} \sum_{i=1}^{N}\left(e_{i, 1}^{2}+e_{i, 2}^{2}\right) \\
& c_{i, 1}^{T} z=w_{1}^{T} \varphi_{1}\left(x_{i}\right)+e_{i, 1} \quad \forall i=1, \ldots, N \\
& c_{i, 2}^{T} z=w_{2}^{T} \varphi_{2}\left(x_{i}\right)+e_{i, 2} \quad \forall i=1, \ldots, N .
\end{aligned}
$$

The solution to this problem can be viewed as a regularized version of (6) in the following sense.

Lemma 1: The solution to the problem (12) is given by the eigenvalue problem

$$
\begin{array}{r}
\left(R+V_{1}\left(\frac{1}{\nu} \Omega_{1}+\frac{1}{\eta} I\right)^{-1} V_{1}^{T}+V_{2}\left(\frac{1}{\nu} \Omega_{2}+\frac{1}{\eta} I\right)^{-1} V_{2}^{T}\right) z \\
=\gamma z \quad(13)
\end{array}
$$

with $R=(I-P)^{T}(I-P)$ and kernel matrices $\Omega_{1}, \Omega_{2} \in$ $\mathbb{R}^{N \times N}$ with $i j$ th entries $\Omega_{1, i j}=K_{1}\left(x_{i}, x_{j}\right)=\varphi_{1}\left(x_{i}\right)^{T} \varphi_{1}\left(x_{j}\right)$, $\Omega_{2, i j}=K_{2}\left(x_{i}, x_{j}\right)=\varphi_{2}\left(x_{i}\right)^{T} \varphi_{2}\left(x_{j}\right)$ for $i, j=1, \ldots, N$ and positive-definite kernel functions $K_{1}(\cdot, \cdot), K_{2}(\cdot, \cdot)$. The matrices $V_{1}, V_{2}$ equal

$$
\begin{aligned}
& V_{1}=\left[c_{1,1} c_{2,1} \ldots c_{N, 1}\right] \\
& V_{2}=\left[c_{1,2} c_{2,2} \ldots c_{N, 2}\right]
\end{aligned}
$$

with elements $c_{i, l}$ as defined in (2).

Proof: See the Appendix

Corollary 1: The dual representations of the model, evaluated at a point $x^{*} \in \mathbb{R}^{p}$, are expressed in terms of the Lagrange multipliers $\alpha_{i, 1}, \alpha_{i, 2}$ [corresponding to the constraints in (12)]

$$
\begin{aligned}
& \hat{z}_{*, 1}=\frac{1}{\nu} \sum_{i=1}^{N} \alpha_{i, 1} K_{1}\left(x_{i}, x^{*}\right) \\
& \hat{z}_{*, 2}=\frac{1}{\nu} \sum_{i=1}^{N} \alpha_{i, 2} K_{2}\left(x_{i}, x^{*}\right)
\end{aligned}
$$

where $\alpha_{i, 1}, \alpha_{i, 2}$ are the unique solution to the linear systems

$$
\left(\frac{1}{\nu} \Omega_{1}+\frac{1}{\eta} I\right) \alpha_{1}=V_{1}^{T} z \quad \text { and } \quad\left(\frac{1}{\nu} \Omega_{2}+\frac{1}{\eta} I\right) \alpha_{2}=V_{2}^{T} z
$$

with $z$ a solution to (13) and $\alpha_{1}=\left[\alpha_{1,1} ; \ldots ; \alpha_{N, 1}\right]$ and $\alpha_{2}=$ $\left[\alpha_{1,2} ; \ldots ; \alpha_{N, 2}\right]$.

The eigenvalue problem (13) has $2 N$ eigenvector solutions corresponding to the eigenvalues $\gamma$. However, it turns out that the selection of the best solution from this pool of $2 N$ candidates is not straightforward. Selection of a best solution interacts in fact with the model selection problem for $\nu$ and $\eta$ and the kernel tuning parameters. The best solution (in terms of good generalization) is often not corresponding with the largest or smallest eigenvalue $\gamma$ in (13). A numerical example to further illustrate this problem will be given in Section VI.

\section{Main Result: Linear System Solution ObTained FROM REFERENCE POINT CONSTRAINTS}

The constrained optimization problem formulations in LS-SVMs enable to incorporate additional constraints in a straightforward manner. We will demonstrate now how adding reference point constraints converts the eigenvalue problem (13) into a new problem where the solution is given by a linear system. We first consider projection of the input data to a 2-D space. Next we consider the general case.

\section{A. Projection to a 2-D Space}

The main idea is to define one of the data points of the training set as a reference point for which the coordinates in the low-dimensional space are chosen (approximately) and fixed beforehand to a nonzero vector. We assume here (without loss of generality) that this is the first data point of the given training set. The new problem formulation becomes

\section{(Problem P2)}

$$
\begin{aligned}
& \min _{z, w_{1}, w_{2}, b_{1}, b_{2}, e_{i, 1}, e_{i, 2}} J_{4}=-\frac{\gamma}{2} z^{T} z+\frac{1}{2}\left(z-P_{D} z\right)^{T}\left(z-P_{D} z\right) \\
& +\frac{\nu}{2}\left(w_{1}^{T} w_{1}+w_{2}^{T} w_{2}\right) \\
& +\frac{\eta}{2} \sum_{i=1}^{N}\left(e_{i, 1}^{2}+e_{i, 2}^{2}\right) \\
& \text { such that } \quad c_{1,1}^{T} z=q_{1}+e_{1,1} \\
& c_{1,2}^{T} z=q_{2}+e_{1,2} \\
& c_{i, 1}^{T} z=w_{1}^{T} \varphi_{1}\left(x_{i}\right)+b_{1}+e_{i, 1} \\
& \forall i=2, \ldots, N \\
& c_{i, 2}^{T} z=w_{2}^{T} \varphi_{2}\left(x_{i}\right)+b_{2}+e_{i, 2} \\
& \forall i=2, \ldots, N \text {. }
\end{aligned}
$$

In this constrained optimization problem, the core part is the LS-SVM mapping of the input data to the coordinates in the 2-D space. The first two terms of the objective function take into account neighborhood preservation. More flexibility is incorporated by modifying (5) into $\sum_{i=1}^{N}\left\|z_{i}-\sum_{j=1}^{N} s_{i j} D z_{j}\right\|_{2}^{2}=$ $\left(z-P_{D} z\right)^{T}\left(z-P_{D} z\right)$ with diagonal matrix $D \in \mathbb{R}^{d \times d}$ and

$$
P_{D}=\left[\begin{array}{cccc}
s_{11} D & s_{12} D & \ldots & s_{1 N} D \\
s_{21} D & s_{22} D & \ldots & s_{2 N} D \\
\vdots & & & \vdots \\
s_{N 1} D & s_{N 2} D & \ldots & s_{N N} D
\end{array}\right] .
$$

For the first training data point $x_{1}$, fictitious coordinates of the nonzero reference point $q=\left[q_{1} ; q_{2}\right] \in \mathbb{R}_{0}^{2}$ are specified by the user. Technically speaking, this point $q$ makes the problem semisupervised instead of unsupervised (in an artificial way). On the other hand, this reference point needs to be sacrificed in the final visualization of the coordinates $z_{i}$. It serves as an eye 
for taking a geometrical perspective on an object. Furthermore, additional bias terms $b_{1}$ and $b_{2}$ are taken in the model. The solution is characterized now as follows.

Lemma 2: Assuming $\gamma \leq 0, \nu, \eta>0$, and $q=\left[q_{1} ; q_{2}\right] \in \mathbb{R}_{0}^{2}$, the unique solution to the problem (17) is given by the linear system

$$
\begin{aligned}
& {\left[\begin{array}{c|c|c}
U & -V_{1} M_{1}^{-1} 1 & -V_{2} M_{2}^{-1} 1 \\
\hline-1^{T} M_{1}^{-1} V_{1}^{T} & 1^{T} M_{1}^{-1} 1 & 0 \\
\hline-1^{T} M_{2}^{-1} V_{2}^{T} & 0 & 1^{T} M_{2}^{-1} 1
\end{array}\right]\left[\begin{array}{c}
z \\
\frac{z}{b_{1}} \\
b_{2}
\end{array}\right]} \\
& =\left[\frac{\eta\left(q_{1} c_{1,1}+q_{2} c_{1,2}\right)}{0}\right]
\end{aligned}
$$

with matrices

$$
\begin{aligned}
U= & \left(I-P_{D}\right)^{T}\left(I-P_{D}\right)-\gamma I+V_{1} M_{1}^{-1} V_{1}^{T} \\
& +V_{2} M_{2}^{-1} V_{2}^{T}+\eta c_{1,1} c_{1,1}^{T}+\eta c_{1,2} c_{1,2}^{T} \\
M_{1}= & \frac{1}{\nu} \Omega_{1}+\frac{1}{\eta} I \quad M_{2}=\frac{1}{\nu} \Omega_{2}+\frac{1}{\eta} I \\
V_{1}= & {\left[c_{2,1} \ldots c_{N, 1}\right] \quad V_{2}=\left[c_{2,2} \ldots c_{N, 2}\right] }
\end{aligned}
$$

and kernel matrices $\Omega_{1}, \Omega_{2} \in \mathbb{R}^{(N-1) \times(N-1)}$ with $i j$ th entries $\Omega_{1, i j}=K_{1}\left(x_{i}, x_{j}\right)=\varphi_{1}\left(x_{i}\right)^{T} \varphi_{1}\left(x_{j}\right)$, $\Omega_{2, i j}=K_{2}\left(x_{i}, x_{j}\right)=\varphi_{2}\left(x_{i}\right)^{T} \varphi_{2}\left(x_{j}\right)$, for $i, j=2, \ldots, N$, and positive-definite kernel functions $K_{1}(\cdot, \cdot), K_{2}(\cdot, \cdot)$.

Proof: See the Appendix

Corollary 2: The primal and dual model representations allow making out-of-sample extensions. Evaluated at point $x^{*} \in \mathbb{R}^{p}$, the predicted coordinates are

$$
\begin{aligned}
& \hat{z}_{*, 1}=w_{1}^{T} \varphi_{1}\left(x^{*}\right)+b_{1}=\frac{1}{\nu} \sum_{i=2}^{N} \alpha_{i, 1} K_{1}\left(x_{i}, x^{*}\right)+b_{1} \\
& \hat{z}_{*, 2}=w_{2}^{T} \varphi_{2}\left(x^{*}\right)+b_{2}=\frac{1}{\nu} \sum_{i=2}^{N} \alpha_{i, 2} K_{2}\left(x_{i}, x^{*}\right)+b_{2}
\end{aligned}
$$

where $b_{1}$ and $b_{2}$ are the solution to (18) and $\alpha_{1}, \alpha_{2} \in \mathbb{R}^{N-1}$ are the unique solutions to the linear systems

$$
M_{1} \alpha_{1}=V_{1}^{T} z-b_{1} 1_{N-1} \quad \text { and } \quad M_{2} \alpha_{2}=V_{2}^{T} z-b_{2} 1_{N-1}
$$

with $z$ the solution to (18) and $\alpha_{1}=\left[\alpha_{2,1} ; \ldots ; \alpha_{N, 1}\right], \alpha_{2}=$ $\left[\alpha_{2,2} ; \ldots ; \alpha_{N, 2}\right]$, and $1_{N-1}=[1 ; 1 ; \ldots, ; 1]$.

The assumption $\gamma \leq 0$ in Lemma 2 is made for establishing convexity of the problem. Observe that in (3) $\gamma>0$ has been assumed. However, this assumption was needed for a different motivation of avoiding the trivial solution and pushing the coordinates away from the origin. In problem $\mathrm{P} 2$, the first term $-\gamma z^{T} z$ has been included for the sake of generality. In practice, $\gamma=0$ can be set, which at the same time will simplify the tuning parameter search process.

\section{B. General Case}

In the general case with dimensionality reduction to a $d$-dimensional space, the problem statement is

\section{(Problem P3)}

$$
\begin{aligned}
& \min _{z, w_{j}, b_{j}, e_{i, j}} J_{5}=-\frac{\gamma}{2} z^{T} z+\frac{1}{2}\left(z-P_{D} z\right)^{T}\left(z-P_{D} z\right) \\
& +\frac{\nu}{2} \sum_{j=1}^{d} w_{j}^{T} w_{j}+\frac{\eta}{2} \sum_{i=1}^{N} \sum_{j=1}^{d} e_{i, j}^{2} \\
& \text { such that } \quad c_{1, j}^{T} z=q_{j}+e_{1, j} \quad \forall j=1, \ldots, d \\
& c_{i, j}^{T} z=w_{j}^{T} \varphi_{j}\left(x_{i}\right)+b_{j}+e_{i, j} \\
& \forall i=2, \ldots, N, \quad j=1, \ldots, d .
\end{aligned}
$$

The solution is characterized in a similar way as in the 2-D case.

Lemma 3: Assuming $\gamma \leq 0, \nu, \eta>0$, and $q \in \mathbb{R}_{0}^{d}$, the unique solution to the problem (21) is given by the linear system

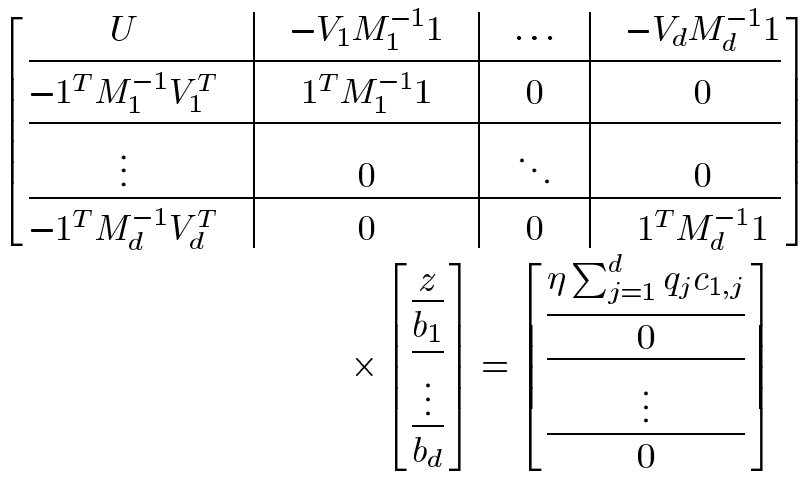

with

$$
\begin{aligned}
& U=\left(I-P_{D}\right)^{T}\left(I-P_{D}\right)-\gamma I \\
&+\sum_{j=1}^{d} V_{j} M_{j}^{-1} V_{j}^{T}+\eta \sum_{j=1}^{d} c_{1, j} c_{1, j}^{T} \\
& M_{j}=\frac{1}{\nu} \Omega_{j}+\frac{1}{\eta} I, V_{j}=\left[c_{2, j} \ldots c_{N, j}\right], \quad j=1, \ldots, d
\end{aligned}
$$

and kernel matrices $\Omega_{j} \in \mathbb{R}^{(N-1) \times(N-1)}$ with $i j$ th entries $\Omega_{j, k l}=K_{j}\left(x_{k}, x_{l}\right)=\varphi_{j}\left(x_{k}\right)^{T} \varphi_{j}\left(x_{l}\right)$, for $j=1, \ldots, d$ and $k, l=2, \ldots, N$, and positive-definite kernel functions $K_{j}(\cdot, \cdot)$.

Proof: See the Appendix

Corollary 3: The primal and dual model representations allow to make out-of-sample extensions. Evaluated at point $x^{*} \in \mathbb{R}^{p}$, the predicted coordinates are

$$
\hat{z}_{*, j}=w_{j}^{T} \varphi_{j}\left(x^{*}\right)+b_{j}=\frac{1}{\nu} \sum_{i=2}^{N} \alpha_{i, j} K_{j}\left(x_{i}, x^{*}\right)+b_{j}
$$

where the bias terms $b_{j}$ are the solution to (22) and $\alpha_{j} \in \mathbb{R}^{N-1}$ are the unique solutions to the linear systems

$$
M_{j} \alpha_{j}=V_{j}^{T} z-b_{j} 1_{N-1}
$$


with $z$ the solution to (22) and $\alpha_{j}=\left[\alpha_{2, j} ; \ldots ; \alpha_{N, j}\right]$ for $j=$ $1, \ldots, d$.

For moderate size data sets, the linear systems can be solved using direct methods [12]. For larger scale problems, iterative solvers such as conjugate gradient methods can be used [12]. However, for conjugate gradient methods, the matrix of the problem needs to be positive definite. The problems (18) and (22) can be converted then into problems with a positive-definite matrix [26], similar as for LS-SVM models in classification and regression with bias terms. The computational complexity is well understood in terms of the condition number of the matrix and the required precision [22].

\section{Model Selection by Validation}

\section{A. Criteria}

To achieve a good generalization ability of the kernel map approach, a careful selection of the tuning parameters is needed. We investigate here two criteria with use of a validation set or cross validation. The kernel map with reference point approach can be tuned then in a similar fashion as kernel-based regression and classification methods with linear system solving, though for a problem of unsupervised instead of supervised learning now. While in problems of regression and classification one aims at designing an accurate predictive model, for data visualization applications, finding a good range of the tuning parameters will usually be satisfactory. One may typically search over a rougher grid for data visualization than in regression and classification applications.

Consider an input training data set $\mathcal{D}_{T}=\left\{x_{i}\right\}_{i=1}^{N}$, validation set $\mathcal{D}_{V}=\left\{x_{j}^{\mathrm{val}}\right\}_{j=1}^{N_{V}}$, and test set $\mathcal{D}_{E}=\left\{x_{l}^{\text {test }}\right\}_{l=1}^{N_{E}}$, where $N$, $N_{V}$, and $N_{E}$ denote the number of training, validation, and test data points, respectively. The following two criteria are considered here with use of a validation set $\mathcal{D}_{V}$ :

- normalized metric multidimensional scaling criterion (normalized metric MDS)

$\min _{\Theta} \mathcal{Q}_{\mathrm{nmds}}=\sum_{i, j=1}^{N_{V}}\left(\frac{\hat{z}_{i}^{\mathrm{val} T} \hat{z}_{j}^{\mathrm{val}}}{\left\|\hat{z}_{i}^{\mathrm{val}}\right\|_{2}\left\|\hat{z}_{j}^{\mathrm{val}}\right\|_{2}}-\frac{x_{i}^{\mathrm{val}} x_{j}^{\mathrm{val}}}{\left\|x_{i}^{\mathrm{val}}\right\|_{2}\left\|x_{j}^{\mathrm{val}}\right\|_{2}}\right)^{2} ;$

- normalized locally linear embedding criterion (normalized LLE)

$$
\min _{\Theta} \mathcal{Q}_{\text {nlle }}=\frac{\sum_{i=1}^{N_{V}}\left\|\hat{z}_{i}^{\mathrm{val}}-\sum_{j=1}^{N_{V}} s_{i j}^{\mathrm{val}} D \hat{z}_{j}^{\mathrm{val}}\right\|_{2}^{2}}{\sum_{j=1}^{N_{V}}\left\|\hat{z}_{j}^{\mathrm{val}}\right\|_{2}^{2}}
$$

with $s_{i j}^{\mathrm{val}}=\exp \left(-\left\|x_{i}^{\mathrm{val}}-x_{j}^{\mathrm{val}}\right\|_{2}^{2} / \sigma^{2}\right)$ denoting the $i j$ th entry of matrix $S^{\mathrm{val}}$;

where $\Theta$ denotes the set of tuning parameters to be determined. Both criteria $\mathcal{Q}_{\text {nmds }}$ and $\mathcal{Q}_{\text {nlle }}$ are normalized in the sense that they are invariant with respect to scaling of the estimated coordinates $\hat{z}_{j}^{\mathrm{val}}$ by a constant. Also in [29], such a type of invariance has been considered at the training level in supervised graph inference. Taking only the numerator part of $\mathcal{Q}_{\text {nlle }}$ turns out to be nonsuitable for model selection purposes.

\section{B. Choice of the Tuning Parameters for Kernel Maps With a Reference Point}

While the coordinates $z$, the support values $\alpha$, and the bias terms $b$ follow from solving linear systems, the choice of a number of tuning parameters needs to be decided. In setting these tuning parameters, on the one hand, there should be enough flexibility to obtain a model that yields good generalization on data sets in different problems. On the other hand, having too many tuning parameters severely complicates the model selection process. For this reason, the following tuning parameters will be fixed beforehand throughout this paper.

- Choice of the reference point $q$ : The choice of the point $q$ plays the role of choosing a geometric perspective on an object. For 2-D and 3-D projections, we take as candidate reference points $q \in \mathcal{S}_{\text {ref }}^{2 \mathrm{D}}$ and $q \in \mathcal{S}_{\text {ref }}^{3 \mathrm{D}}$, respectively, where

$$
\begin{aligned}
& \mathcal{S}_{\mathrm{ref}}^{2 \mathrm{D}}=\{[+1 ;+1][+1 ;-1][-1 ;+1][-1,-1]\} \\
& \mathcal{S}_{\mathrm{ref}}^{3 \mathrm{D}}=\{[+1 ;+1 ;+1][+1 ;-1 ;+1][-1 ;+1 ;+1] \\
& {[-1 ;-1 ;+1][+1 ;+1 ;-1][+1 ;-1 ;-1] } \\
& {[-1 ;+1 ;-1][-1,-1 ;-1]\} . }
\end{aligned}
$$

The role of this reference point is further illustrated by examples in Section VI. A multiplication of the reference vector $q$ with a positive constant $c \in \mathbb{R}^{+}$leads to similar results as taking this constant $c=1$, corresponding to the choices in (27).

- Choice of the diagonal matrix D: Experiments indicate that better results are obtained by taking the diagonal matrix $D$ different from the identity matrix. Throughout this paper, for 2-D projection, we fix $D=\operatorname{diag}\{10,1\}$, and for 3-D projections, $D=\operatorname{diag}\{10,5,1\}$.

- Choice of $\gamma$ : As explained in Section IV, we set $\gamma=0$.

In this way, the unknown set of tuning parameters $\Theta$ is reduced to the following.

- Kernels tuning parameters in $s_{i j}, K_{1}, K_{2},\left(K_{3}\right)$ : When using radial basis functions in

$$
\begin{aligned}
s_{i j} & =\exp \left(-\left\|x_{i}-x_{j}\right\|_{2}^{2} / \sigma^{2}\right) \\
K_{j}(x, z) & =\exp \left(-\|x-z\|_{2}^{2} / \sigma_{j}^{2}\right)
\end{aligned}
$$

for $j=1, \ldots, d$, the kernel parameters $\sigma, \sigma_{j}$ need to be determined. Especially the determination of a good range for these values turns out to be relevant as well as the values relative with respect to each other.

- Regularization constants $\nu, \eta$ : The determination of a good range is needed for the value $\nu$. The value $\eta=1$ is set.

It is also recommended to (linearly) scale the input data properly with elements of the input data in the range $[-1,+1]$ before applying the method or to normalize the data.

\section{Algorithms: Randomized Validation Set and Cross Validation}

Data visualization techniques are frequently applied for exploratory data analysis. This process is more interactive than the design of models for regression or classification purposes. 
For existing methods, one often varies then the tuning parameters of the method that results in different data visualizations of a given data set. Currently, due to the lack of general theories for setting the tuning parameters, this model selection aspect is mostly ignored, often resulting in a quite subjective data visualization process.

One aim of this paper is to make this data visualization process less subjective by estimating an underlying model that one can extend and evaluate on validation data. We describe now two practical algorithms: a cross-validation method with grid search and a method with use of a randomized validation set that is more suitable for interactive data exploration. The following cross-validation algorithm is proposed.

Algorithm 1: Cross validation with grid search for 3-D visualization

1. Input: load data set $\mathcal{D}$, linearly scale elements to interval $[-1,+1]$.

2. Partition $\mathcal{D}$ into test part $\mathcal{D}_{E}$ and part $\mathcal{D}_{T V}$ for training-validation with cross-validation (e.g. $n_{C V}=10$ for tenfold cross validation).

3. Assign the first data point of $\mathcal{D}_{T V}$ to be the reference point (kept the same over all folds in the cross-validation process) and choose $q \in \mathcal{S}_{\text {ref }}^{3 \mathrm{D}}$ for (27).

4. Define tuning parameter grid

$$
\begin{aligned}
& v_{\sigma^{2}}=p \times[10 ; 20 ; 30 ; 40 ; 50 ; 60 ; 70 ; 100 ; 120] \in \mathbb{R}^{n_{\sigma^{2}}} \\
& v_{\sigma_{1}^{2}}=p \times[10 ; 20 ; 30 ; 40 ; 50 ; 60 ; 70 ; 100 ; 120] \in \mathbb{R}^{n_{\sigma_{1}^{2}},} \text { for }(28) \\
& v_{\nu}=[0.1 ; 1 ; 10] \in \mathbb{R}^{n_{\nu}} \\
& \sigma_{2}^{2}=a_{2} \sigma_{1}^{2}, \sigma_{3}^{2}=a_{3} \sigma_{1}^{2}, \quad \text { where } a_{2}, a_{3} \in\{0.5,1,2\} .
\end{aligned}
$$

5. For CV-loop index $=1$ to $n_{C V}$

6. Split $\mathcal{D}_{T V}$ into training part $\mathcal{D}_{T}$ and validation part $\mathcal{D}_{V}$ according to the cross-validation procedure.

7. For tuning loops over indices $=1$ to $\left\{n_{\sigma^{2}}, n_{\sigma_{1}^{2}}, n_{\nu}, 3,3\right\}$

8. Construct kernel matrices $S, S^{\mathrm{val}}, \Omega_{1}, \Omega_{2}, \Omega_{3}$. Construct matrices $P_{D}, V_{1}, V_{2}, V_{3}$.

9. Solve linear system (22) that gives $z, b_{j}$. Solve linear system (24) that gives $\alpha_{j}$ of the model.

10. Evaluate the model on validation data based on (23) to obtain $\hat{z}_{j}^{\mathrm{val}}$.

11. Compute the criteria $\mathcal{Q}_{\text {nmds }}$ and $\mathcal{Q}_{\text {nlle }}$ on validation data (25) and (26).

12. End tuning loops

13. End CV loop

14. Output: Sort results according to $\mathcal{Q}_{\text {nmds }}, \mathcal{Q}_{\text {nlle }}$. Visualize data for $\mathcal{D}_{E}$ and $\mathcal{D}_{T V}$.

Note that in $v_{\sigma^{2}}, v_{\sigma_{1}^{2}}$ a scaling with $p$ is taken. For higher dimensional input spaces, the $\sigma^{2}, \sigma_{1}^{2}$ values need to scale proportionally. It could also be needed to enlarge or further refine the grid depending on the outcome of the grid search. In principle, one could also define vectors $v_{\sigma_{2}^{2}}, v_{\sigma_{3}^{2}}$ similar to $v_{\sigma_{1}^{2}}$. However, this would lead to a very time-consuming search process over all different tuning parameters. Experiments also indicate that for a good generalization the ranges of $\sigma, \sigma_{1}, \sigma_{2}, \sigma_{3}$ should not be too far away from each other. This motivates the search over neighboring ranges for $\sigma_{2}, \sigma_{3}$ relative with respect to $\sigma_{1}$.

Cross validation with grid search is especially popular for kernel-based methods in regression and classification problems. However, for exploratory data analysis, a more interactive search process might be more appealing to the user. The use of a single validation set is one possibility but the tuning might become too specific with respect to this validation set. Therefore, tuning parameter search with a randomized validation set is proposed to make the result less depending on a specific set and keep the overall search process sufficiently interactive. No grid is defined in this case. The user interactively explores the tuning parameter search space then trying to discover structure in the data. This process can be guided now by the criteria $\mathcal{Q}_{\text {nmds }}$ and $\mathcal{Q}_{\text {nlle }}$ evaluated on validation data. This gives an indication to the user about whether the observed structure can be trusted in an objective way (in the sense of good generalization) or is rather a "fake" structure corresponding to a situation of data overfitting. Experiments also indicate that the criterion $\mathcal{Q}_{\text {nlle }}$ has a tendency to overfit for small values of $\sigma, \sigma_{1}, \sigma_{2}, \sigma_{3}$. The following algorithm is proposed for use of a randomized validation set.

Algorithm 2: Randomized validation set for 3-D visualization

1. Input: load data set $\mathcal{D}$, linearly scale elements to interval $[-1,+1]$.

2. Partition $\mathcal{D}$ into test part $\mathcal{D}_{E}$ and part $\mathcal{D}_{T V}$ for training validation.

3. Assign the first data point of $\mathcal{D}_{T V}$ to be the reference point (kept the same over all folds in the cross-validation process) and choose $q \in \mathcal{S}_{\text {ref }}^{3 \mathrm{D}}$ in (27).

4. Decide on the numbers $N, N_{V}$ for splitting $\mathcal{D}_{T V}$ into training and validation parts.

Decide on the number of randomizations $n_{\text {rand }}$ of the validation set.

5. Make a choice of the tuning parameters $\sigma, \sigma_{1}, \sigma_{2}, \sigma_{3}, \nu$.

6. For randomization loop, index $=1$ to $n_{\text {rand }}$

7. Randomly split $\mathcal{D}_{T V}$ into training part $\mathcal{D}_{T}$ and validation part $\mathcal{D}_{V}$

8. Construct kernel matrices $S, S^{\text {val }}, \Omega_{1}, \Omega_{2}, \Omega_{3}$. Construct matrices $P_{D}, V_{1}, V_{2}, V_{3}$.

9. Solve linear system (22) that gives $z, b_{j}$. Solve linear system (24) that gives $\alpha_{j}$ of the model.

10. Evaluate the model on validation data based on (23) to obtain $\hat{z}_{j}^{\mathrm{val}}$.

11. Compute the criteria $\mathcal{Q}_{\text {nmds }}$ and $\mathcal{Q}_{\text {nlle }}$ on validation data (25) and (26).

12. End randomization loop

13. Compute the means of $\mathcal{Q}_{\text {nmds }}$ and $\mathcal{Q}_{\text {nlle }}$ over the $n_{\text {rand }}$ randomized validation sets. 


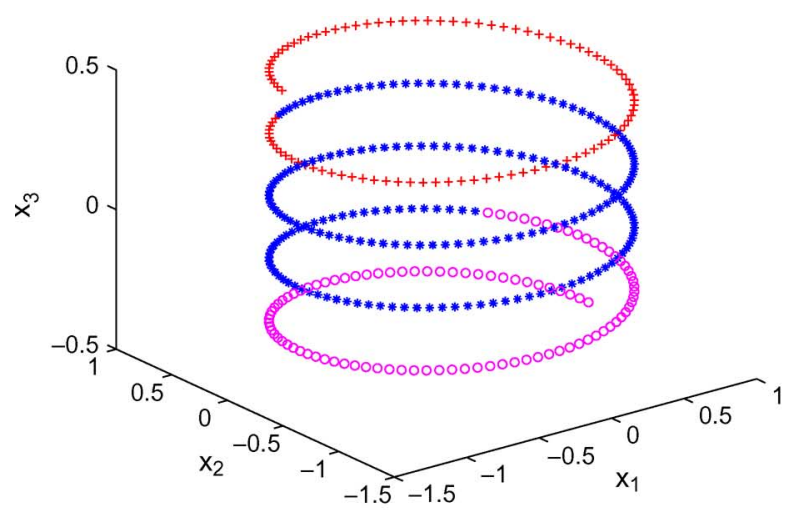

(a)

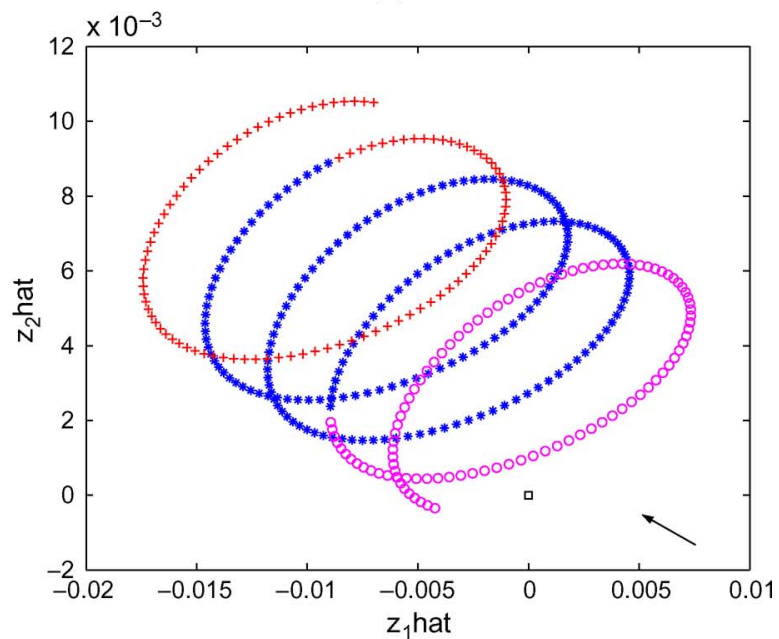

(b)

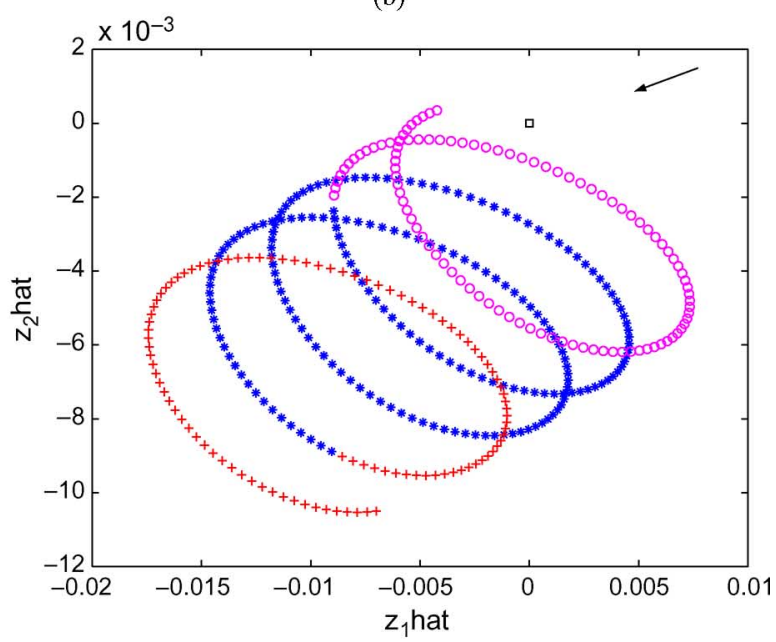

(c)

Fig. 1. (a) Given input data of a spiral data set in a 3-D space [training data (blue $*$ ), validation data (magenta o), test data $($ red +$)]$. (b) Kernel map with a reference point $[+1 ;-1]$. (c) Kernel map with a reference point $[+1 ;+1]$. The positions of the reference points are indicated by the arrows. The tuning parameters are based on the use of a validation set. The method achieves good generalization on test data.

14. Interactive output: Visualize data for $\mathcal{D}_{E}$ and $\mathcal{D}_{T V}$ for the chosen tuning parameters.

15. If validation criteria indicate a good solution, End search

Else go to 5 and continue the search process.
Algorithms 1 and 2 have been described here for the 3-D visualization case. The algorithms for $2-\mathrm{D}$ visualization are similar but simpler as one has the search over fewer tuning parameters. Under supplementary material, a demo is provided to illustrate the method with use of a single validation set and the criterion $\mathcal{Q}_{\text {nmds }}$.

\section{EXAMPLES}

\section{A. Spiral Problem}

1) Role of the Reference Point: In this first toy problem, a spiral data problem is considered with data generated in Matlab, ${ }^{2}$ scaled to ranges $[-1,1]$ and made zero mean. The given input data are in a 3-D space, shown in Fig. 1. The problem P2 was considered for data visualization in 2-D. The figure illustrates the role of the reference point with different choices of $q \in \mathcal{S}_{\text {ref }}^{2-D}$ from (27). Different choices of $q$ lead to different perspectives on the object. In this example, the tuning parameter selection was done interactively with use of a single validation set resulting into the kernel parameters $\sigma^{2}=100, \sigma_{1}^{2}=80, \sigma_{2}^{2}=0.1 \sigma_{1}^{2}$, and $\nu=0.5$. All other tuning parameters were taken as described in Section V. Besides good visualization of the training and validation set parts, an excellent generalization is also obtained on test data. The arrows indicate the positions of the reference points, which are located outside the range of all other points, playing the role of an eye for looking at the object.

2) Tuning by Cross Validation: In Figs. 2 and 3, the results of tuning parameter selection by tenfold cross validation is illustrated with application of Algorithm 1 for 2-D visualization. Fig. 2 shows the two best results for the considered (rough) grid according to the criteria $\mathcal{Q}_{\text {nmds }}$ and $\mathcal{Q}_{\text {nlle }}$. The reference point $q=[+1 ;-1]$ is chosen. The grid search process with evaluated criteria over the ten different runs is shown in Fig. 3. The resulting tuning parameters are $\sigma^{2}=210, \sigma_{1}^{2}=210, \sigma_{2}^{2}=105$, and $\nu=1$ according to $\mathcal{Q}_{\text {nmds }}$ and $\sigma^{2}=300, \sigma_{1}^{2}=210$, $\sigma_{2}^{2}=105$, and $\nu=0.1$ according to $\mathcal{Q}_{\text {nlle }}$. For smaller values of $\sigma, \sigma_{1}, \sigma_{2}$ (not included in this grid search), it can be observed that the criterion $\mathcal{Q}_{\text {nlle }}$ has a tendency to overfit. The mean value over the different runs becomes small then, but with a large variance over the ten different runs (such that one can detect the problem in this way).

3) Comparison with Other Methods: A comparison with the following other methods is shown in Fig. 4: MDS, PCA, LLE (Hessian LLE, which is not shown, gives a qualitatively similar result to LLE), Laplacian eigenmaps, and diffusion maps. ${ }^{3}$ In this case, no division is made in training, validation, and test parts. From the total of 800 data points 600 points were randomly selected for the training with these methods. The

\footnotetext{
${ }^{2}$ Spiral data Matlab code: $t=0.01: 0.01: 4 ; x=\cos \left(7^{*} t\right) ; y=\sin \left(7^{*} t\right)$; $z=t$.

${ }^{3}$ For comparison with other methods including MDS, PCA, ISOMAP, LLE, Hessian LLE, Laplacian, diffusion map, LTSA: http://www.math.umn.edu/ $\sim$ wittman/mani/, http://www.cs.toronto.edu/ roweis/lle/cpde/html
} 

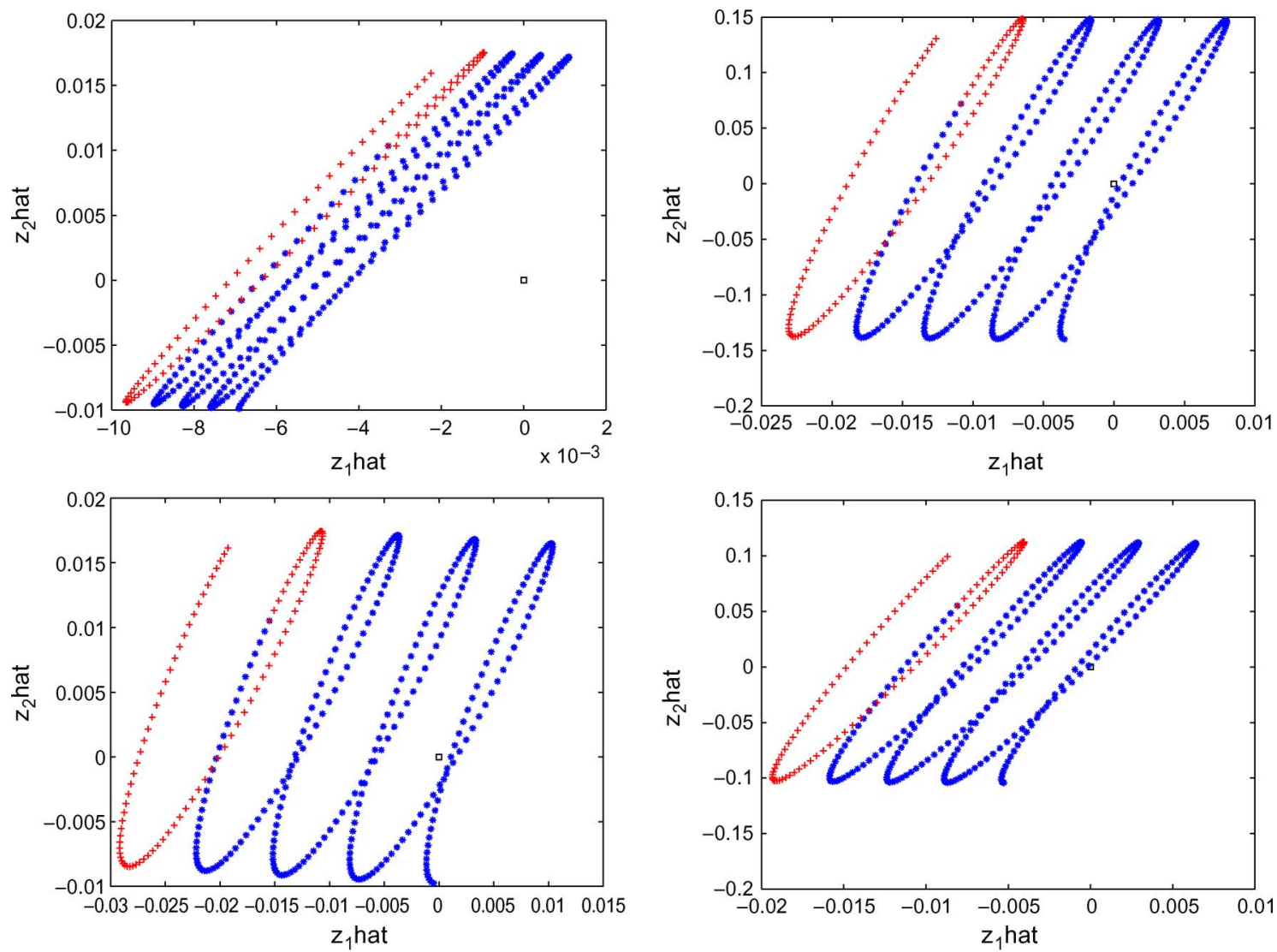

(a)

(b)

Fig. 2. Kernel maps with a reference point: illustration of Algorithm 1 using a tenfold cross-validation procedure on the spiral data. (a) Two best results selected according to criterion $\mathcal{Q}_{\text {nmds }}$. (b) Two best results according to criterion $\mathcal{Q}_{\text {nlle }}$.

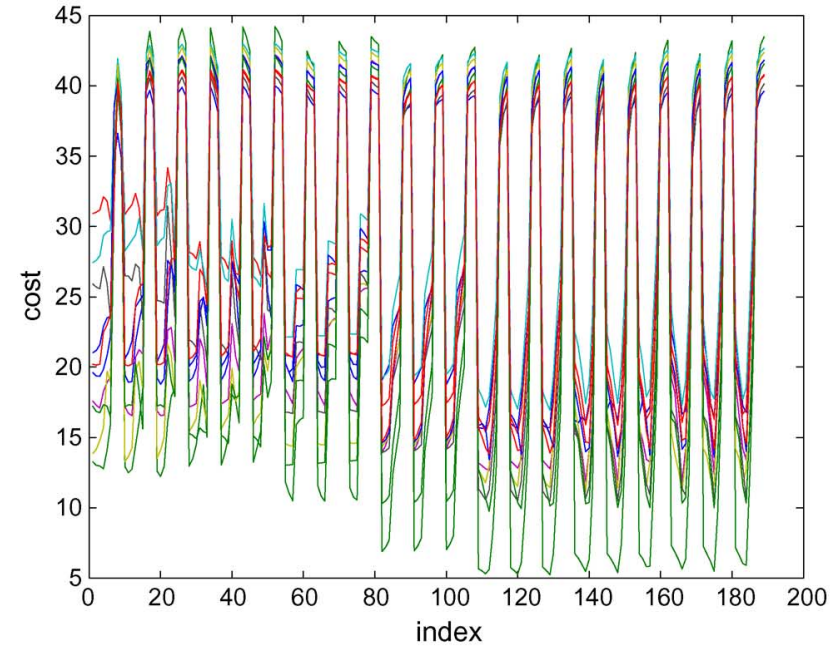

(a)

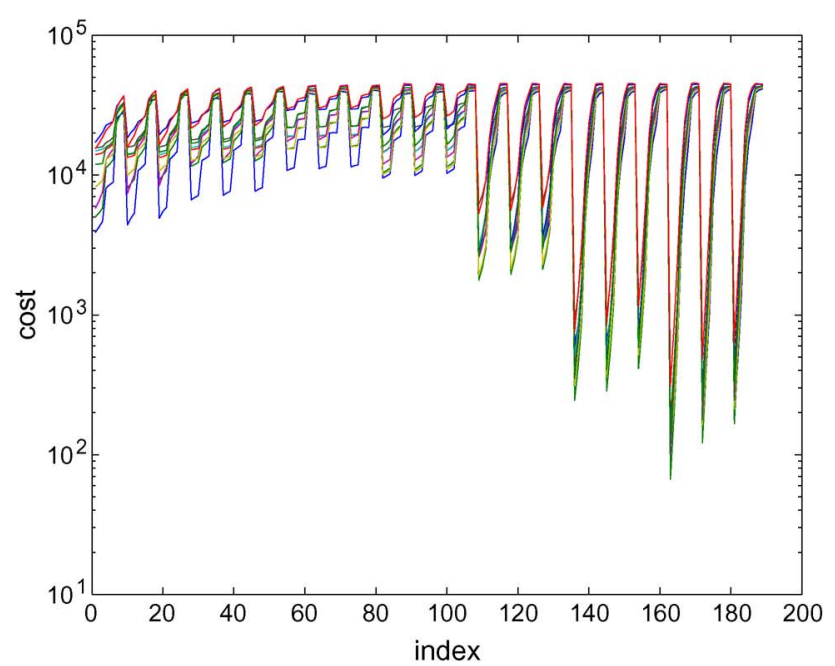

(b)

Fig. 3. Algorithm 1 with tenfold cross validation: (a) $\mathcal{Q}_{\text {nmds }}$ criterion with respect to a running index for a grid search process; (b) $\mathcal{Q}_{\text {nlle }}$ criterion with respect to the running index. The different curves on both plots correspond to the ten runs in the tenfold cross validation.

tuning parameters are set as follows: (LLE and Hessian LLE) ten nearest neighbors, (Laplacian eigenmap) ten nearest neighbors, sigma $=10$, (diffusion map) sigma $=10$, alpha $=1$. The results of LLE and Hessian LLE are closest to the kernel map with reference point method.
4) Comparison with Eigenvalue Problem Method: Also the problem P1 with the solution characterized by the eigenvalue problem has been tested. All experiments indicated that the component selection was difficult in that case: the best solutions were often not corresponding to the largest or smallest 


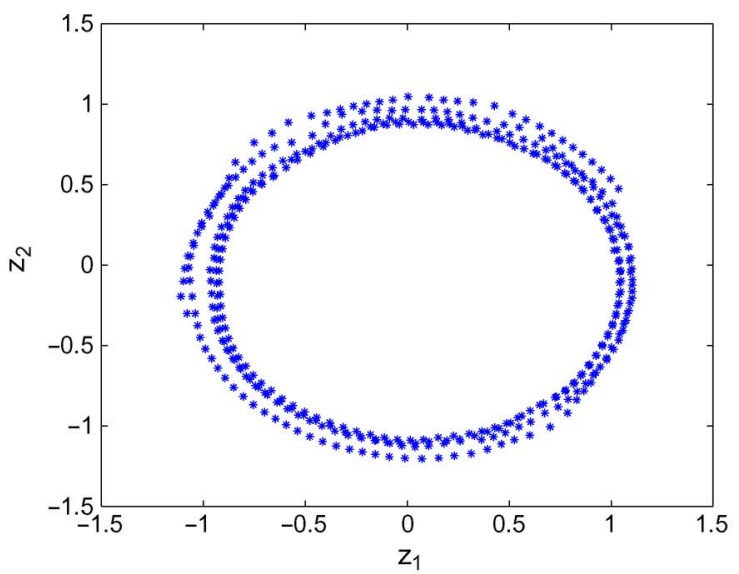

(a)

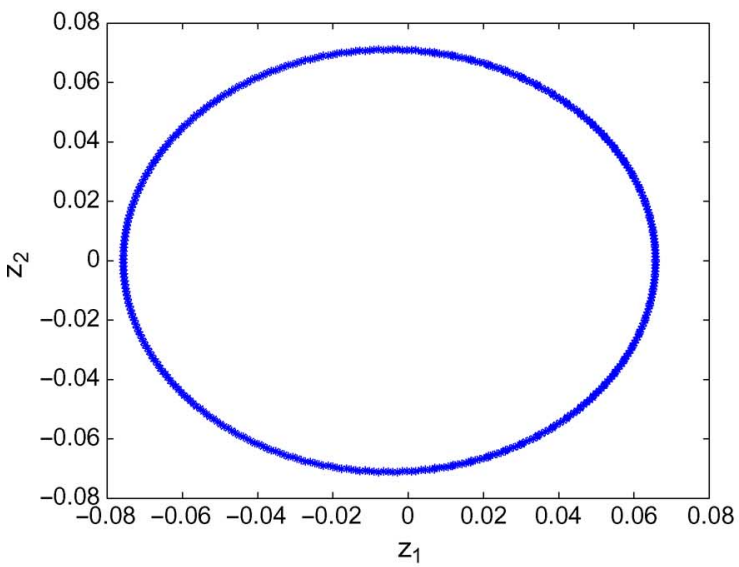

(c)

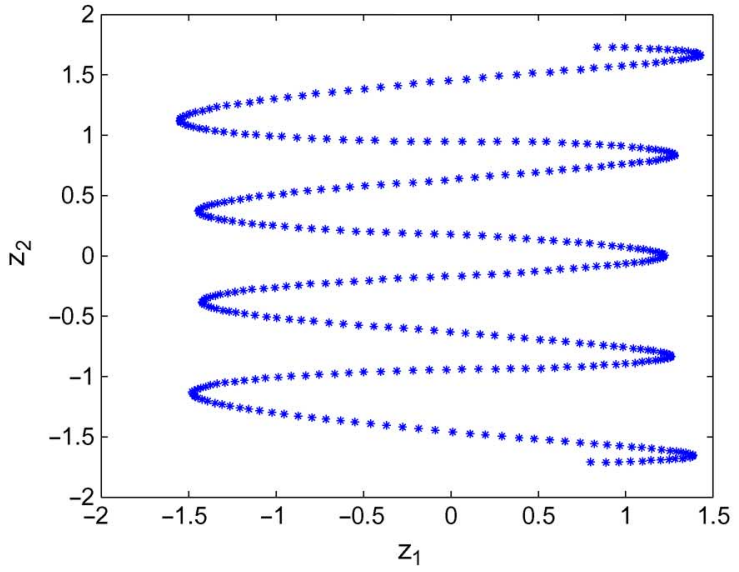

(e)

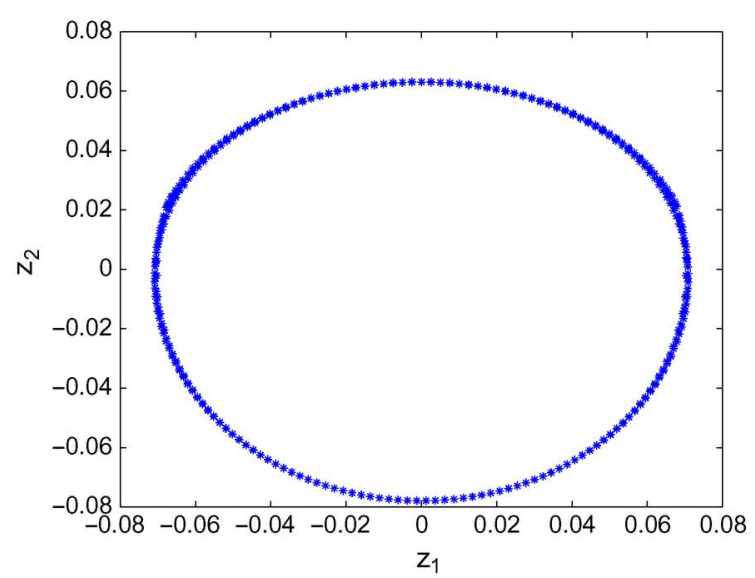

(b)

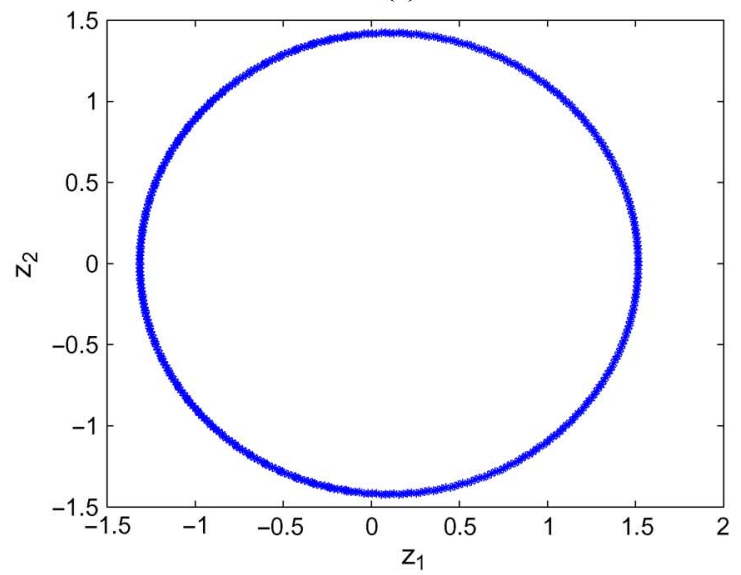

(d)

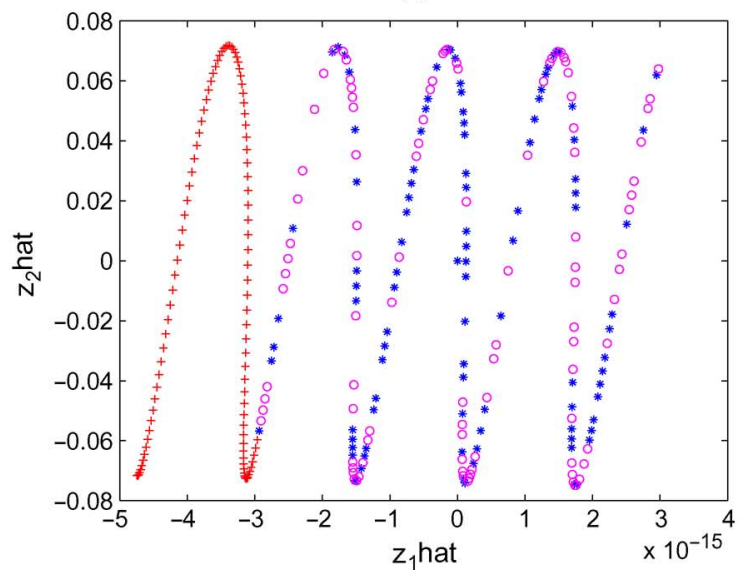

(f)

Fig. 4. Spiral data set: comparison with existing methods. (a) MDS, (b) Laplacian eigenmap, (c) PCA, (d) diffusion map, (e) LLE, and (f) comparison with solution to eigenvalue problem for problem P1, which is numerically ill-conditioned.

eigenvalue in (13). As a result an exhaustive search through different eigenvector solution candidates may be needed then together with a validation procedure for the optimal component selection (or by any other suitable model selection procedure), which is computationally much harder. Moreover, the conditioning of the numerical algorithms for computing the eigenvector solutions is inferior (even if one specifies to compute only a subset of the eigenvectors such as with the Matlab func- tions svds and eigs) with respect to the well-conditioned linear systems to be solved in problems P2 and P3. A numerical example is given in Fig. 4: the optimal component in this case did not correspond to the largest or smallest eigenvalue. Such issues also occur in denoising applications with kernel PCA [19], [24] where the ordering of the eigenvalues and the corresponding components does not always correspond with their relevance towards the reconstruction error. Also note the bad numerical 
conditioning for this problem in Fig. 4(f) with $\hat{z}_{1}$ values in the order of magnitude $10^{-15}$ while $\hat{z}_{2}$ is in the order $10^{-2}$, despite the specification of suitable tolerance parameters.

\section{B. Swiss Roll Data}

A well-known example is the swiss roll problem [16]. We generated the input data in Matlab, ${ }^{4}$ scaled to ranges $[-1,1]$ and made zero mean. The given data in 3-D are shown in Fig. 5. Fig. 5 also shows the result of a kernel map with reference point with use of a single validation set, where the first 100 points are chosen as the validation set and the next 600 as the training data. The resulting tuning parameters for problem $\mathrm{P} 2$ with data visualization in 2-D are $\sigma^{2}=200, \sigma_{1}^{2}=200, \sigma_{2}^{2}=\sigma_{1}^{2}$, and $\nu=0.5$. The reference point $q=[+1 ;-1]$ is chosen.

In Fig. 5(c), tuning parameter search is done using Algorithm 2 with a randomized validation set and $n_{\text {rand }}=3$. The training, validation, and test parts are partitioned as $N=600 \times(2 / 3)$, $N_{V}=600 \times(1 / 3)$, and $N_{E}=200$. The best results indicated by $\mathcal{Q}_{\text {nmds }}$ and $\mathcal{Q}_{\text {nlle }}$ are in the ranges $\sigma^{2} \in[125,150], \sigma_{1}^{2} \in$ [50,100], $\sigma_{2}^{2}=\sigma_{1}^{2}$, and $\nu=1$. Fig. 5(c) shows an obtained result from this selected range of tuning parameters.

A comparison with the following other methods is shown in Fig. 6: MDS, PCA, LLE, Laplacian eigenmaps, and diffusion maps. In this case, no division is made in training, validation, and test parts. All 800 data points are used for the training. The results of the methods LLE and Laplacian eigenmaps give very different results depending on the choice of their tuning parameters. While these methods are often applied in practice with a relatively small number of nearest neighbors (e.g., ten), for achieving results similar to the solution of kernel maps with a reference point, a very large number of nearest neighbors is needed in this case (different from the choices illustrated, e.g., in [2]): (LLE) 200 nearest neighbors, (Laplacian eigenmap) 300 nearest neighbors, and sigma $=50$. For LLE, the significant difference in results between using 50 and 200 nearest neighbors is shown: for smaller numbers of nearest neighbors the rolling structure is not obtained. Larger numbers of nearest neighbors also takes much higher computation times. The diffusion map method is applied with sigma $=10$ and alpha $=1$. The tuning parameters of these existing methods were set in such a way that they reveal a similar structure as the kernel map with reference point method.

\section{Alon Colon Cancer Microarray Data Set}

In this example, we aim at visualizing the gene distribution for the Alon colon cancer microarray data set. ${ }^{5}$ In total 1500 genes are taken: the number of training, validation, and test parts are partitioned as $N=1000 \times(1 / 2), N_{V}=1000 \times(1 / 2)$, and $N_{E}=500$. For the given microarray data set, the dimension of the input space is $p=62$.

${ }^{4}$ Swiss roll data Matlab code: $N_{\text {tot }}=700 ; t t=\left(3^{*} p i / 2\right) *(1+2 *(1: 1$ : $\left.\left.N_{\text {tot }}\right) / N_{\text {tot }}\right) ;$ height $=2 * \operatorname{rand}\left(1, N_{\text {tot }}\right) ; X=\left[t t .^{*} \cos (t t)\right.$; height; $t t . * \sin (t t)]$, http://www.cs.toronto.edu/ roweis/lle/code/swissroll.m

${ }^{5} \mathrm{http} / / /$ hornes.esat.kuleuven.be/ npochet/Bioinformatics/

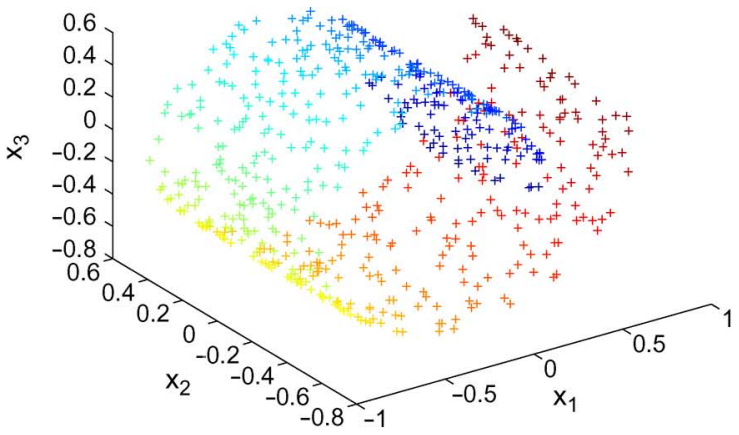

(a)

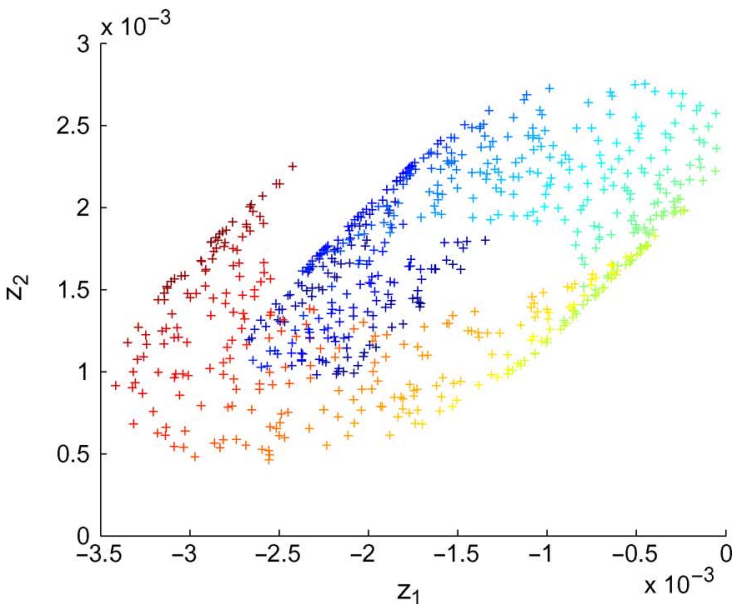

(b)

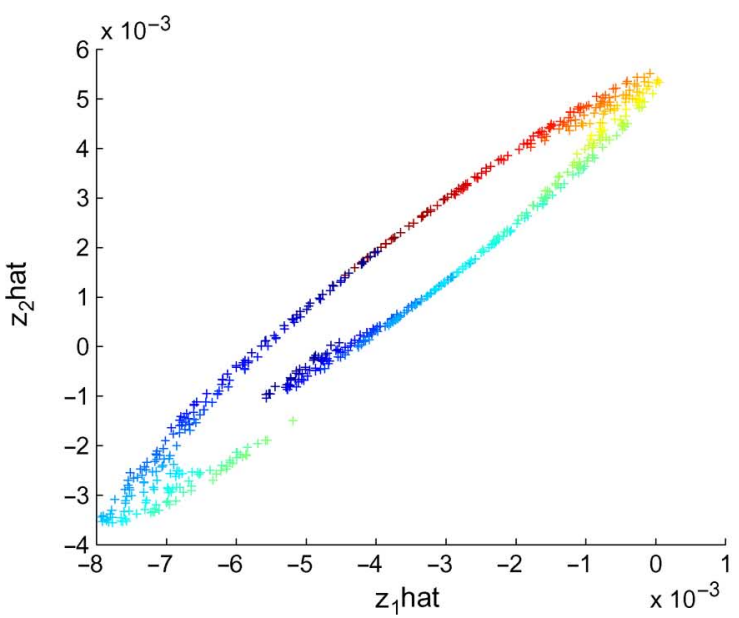

(c)

Fig. 5. (a) Swiss roll data set (containing 600 training data points and 100 validation set points); (b) 2-D visualization with dimensionality reduction using the kernel map with reference point; (c) application of Algorithm 2 with a randomized validation set. The figure shows a result for which the criteria $\mathcal{Q}_{\text {nmds }}$ and $\mathcal{Q}_{\text {nlle }}$ indicate a good generalization performance.

In Fig. 7, the results of 2-D visualization by the kernel map with reference point are shown. Tuning parameter search is done using Algorithm 2 with a randomized validation set and $n_{\text {rand }}=$ 3 . The resulting tuning parameters for problem $\mathrm{P} 2$ with data visualization in 2-D are $\sigma^{2}=10000, \sigma_{1}^{2}=1000, \sigma_{2}^{2}=0.1 \sigma_{1}^{2}$, and $\nu=50$. The reference point $q=[+1 ;-1]$ is chosen. Fig. 7 also shows the result of assigning different parts to be training 


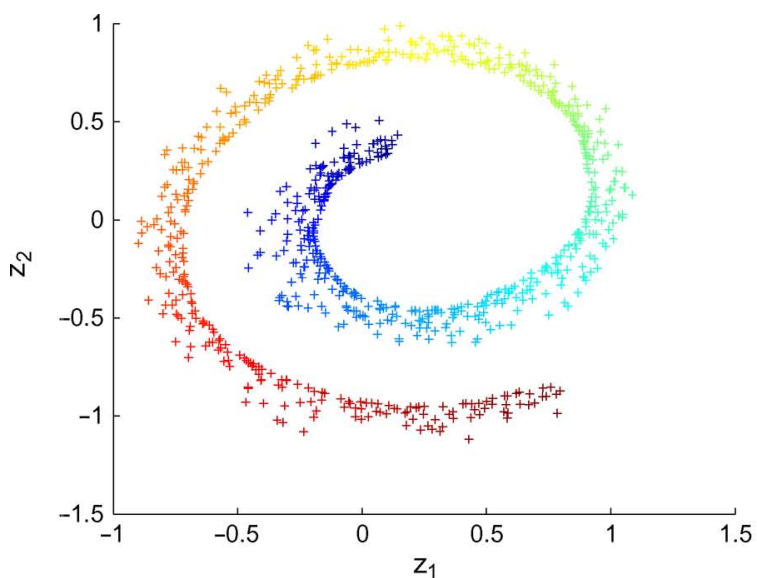

(a)

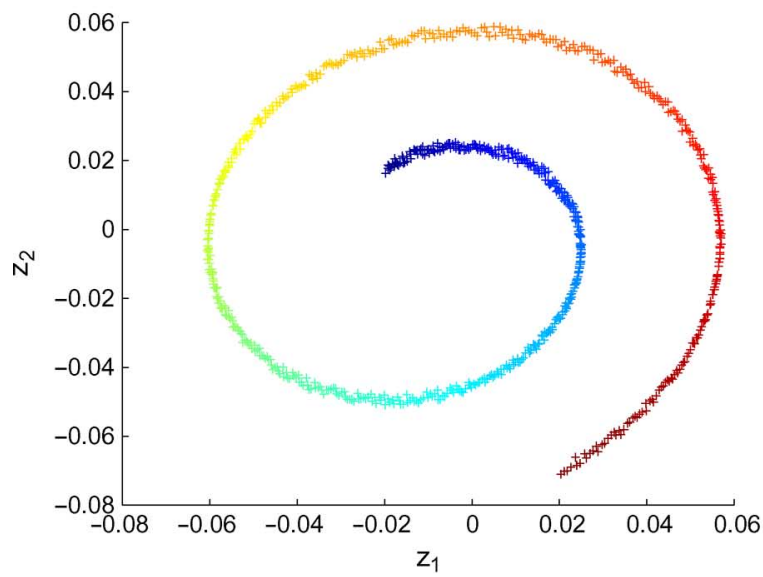

(c)

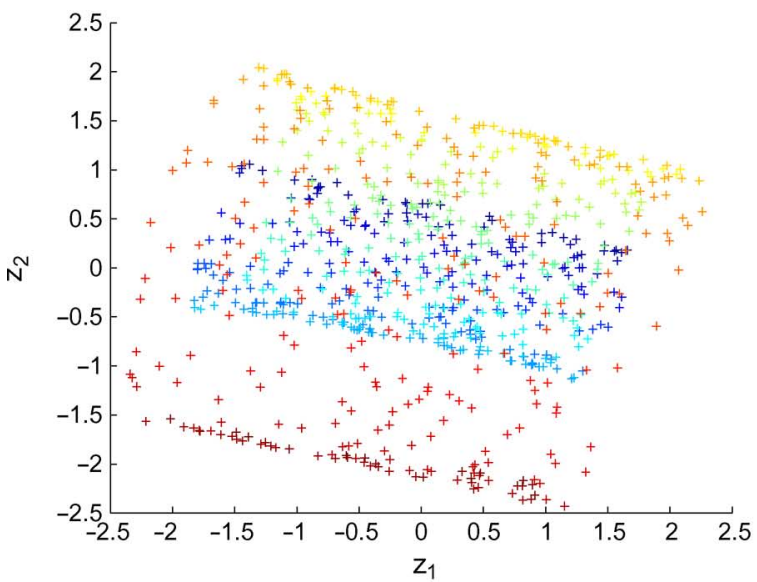

(e)

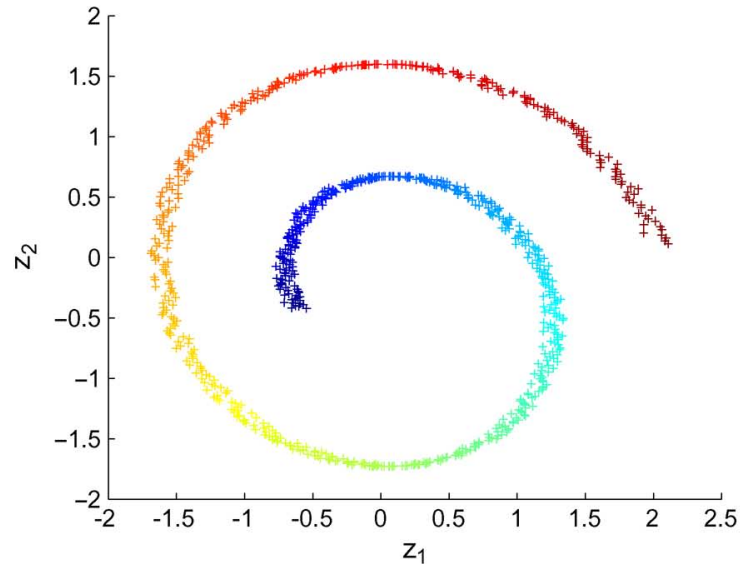

(b)

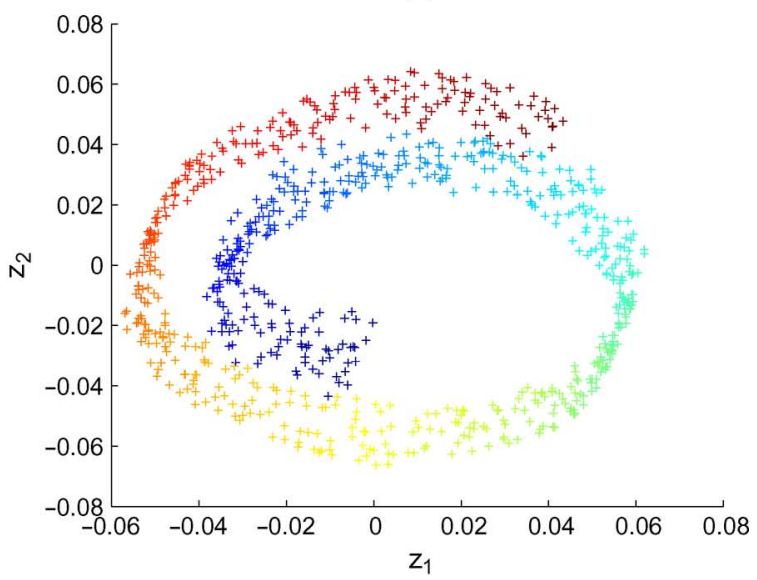

(d)

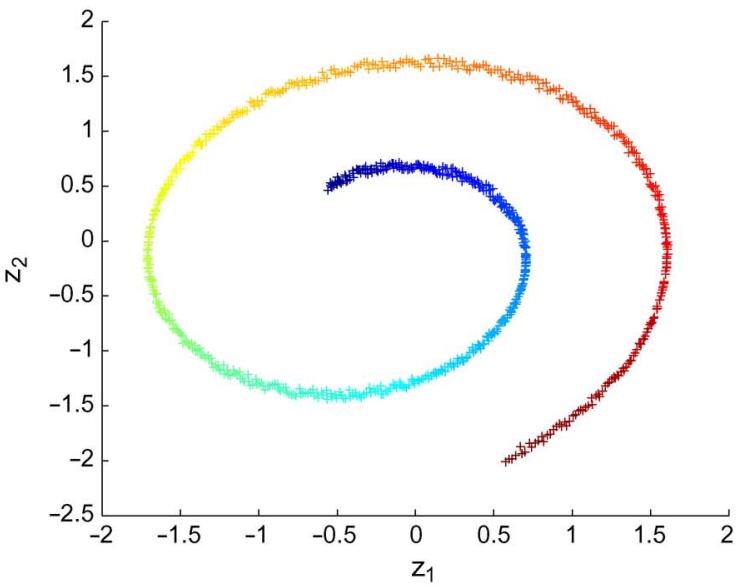

(f)

Fig. 6. Swiss roll data: comparison with existing methods. (a) MDS, (b) LLE with 200 nearest neighbors, (c) PCA, (d) Laplacian eigenmap with 300 nearest neighbors (only a large number reveals the rolling structure), (e) LLE with 50 nearest neighbors, and (f) diffusion map. Tuning parameters were set to reveal similar structure as the kernel map with reference point method.

and test data, which does not affect the discovered structure in the data visualization.

In Fig. 8(a), the role of the reference point is illustrated in 3-D visualization of the data with a choice of $q \in \mathcal{S}_{\text {ref }}^{3-D}$ from (27). Different choices of $q$ lead to different perspectives on the object. The tuning parameter search is done using Algorithm 2 with a randomized validation set and $n_{\text {rand }}=3$. The resulting tuning parameters for problem $\mathrm{P} 3$ with data visualization in 3-D are $\sigma^{2}=10000, \sigma_{1}^{2}=1000, \sigma_{2}^{2}=0.8 \sigma_{1}^{2}, \sigma_{3}^{2}=0.3 \sigma_{1}^{2}$, and $\nu=50$, indicated by the criteria $\mathcal{Q}_{\text {nmds }}$ and $\mathcal{Q}_{\text {nlle }}$.

A comparison with the following other methods is shown in Fig. 8: MDS, PCA, LLE, Laplacian eigenmaps, and diffusion maps. In this case, no division is made in training, validation, and test parts. From the total of 1500 data points 700 points 

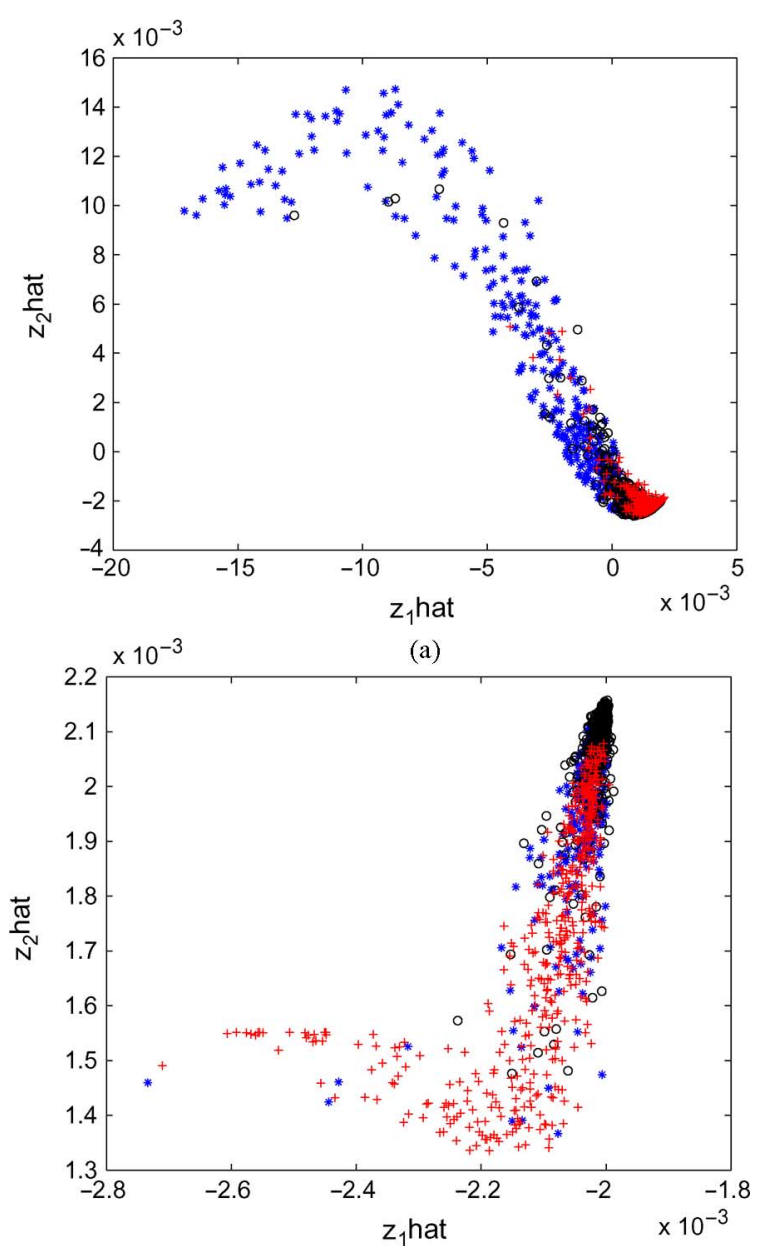

(b)

Fig. 7. Alon colon cancer microarray data set: 2-D visualization of the gene distribution obtained by the kernel map reference point method [training data (blue $*$ ), validation data (black o), test data (red +)]. Both figures show the result for different assignments of training and test parts of the data, which does not affect the obtained structure.

were randomly selected as the training set for these methods. For LLE, Laplacian eigenmaps, and diffusion maps, the tuning parameters were selected that lead to similar structure as found by kernel maps with reference points. For Laplacian eigenmaps, the result is very much outlying with respect to all other methods on this example. The tuning parameters are as follows: (LLE) 100 nearest neighbors, (Laplacian eigenmap) ten nearest neighbors, sigma $=2000,($ diffusion map) sigma $=100$, and alpha $=1$.

\section{Santa Fe Chaotic Laser Data}

In this example, we visualize the time-series $\left\{y_{t}\right\}_{t=1}^{T}$ of the Santa Fe chaotic laser data set ${ }^{6}$ by defining the vectors $y_{t \mid t-m}=$ $\left[y_{t} ; y_{t-1} ; y_{t-2} ; \ldots ; y_{t-m}\right]$ with $m=9$. The data set consists then of the points $\left\{y_{t \mid t-m}\right\}_{t=m+1}^{t=m+N_{\text {tot }}}$ with $N_{V}=600 \times(1 / 3)$ validation data (first part) and $N=600 \times(2 / 3)$ training data points (middle part) and $N_{E}=300$ test data (Fig. 9) in a $p=10$ dimensional input space.

\footnotetext{
${ }^{6}$ Www-psych.stanford.edu/ andreas/Time-Series/SantaFe.html
}

In Fig. 9(b), tuning parameter search is done using Algorithm 2 with a randomized validation set and $n_{\text {rand }}=3$. The best results for 2-D visualization indicated by $\mathcal{Q}_{\text {nmds }}$ and $\mathcal{Q}_{\text {nlle }}$ are $\sigma^{2}=500, \sigma_{1}^{2}=100, \sigma_{2}^{2}=\sigma_{1}^{2}$, and $\nu=10$. The reference point $q=[+1 ;-1]$ is chosen.

In Fig. 10(a), a 3-D visualization is shown for a reference point choice. The application of Algorithm 2 with a randomized validation set leads to the selection of the tuning parameters as $\sigma^{2}=480, \sigma_{1}^{2}=120, \sigma_{2}^{2}=\sigma_{1}^{2}, \sigma_{3}^{2}=\sigma_{1}^{2}$, and $\nu=10$.

A comparison with the following other methods is shown in Fig. 10: MDS, PCA, LLE, Laplacian eigenmaps, and diffusion maps. In this case, no division is made in training, validation, and test parts. All 900 data points were used as training set for these methods. The tuning parameters are as follows: (LLE) 100 nearest neighbors, (Laplacian eigenmap) 300 nearest neighbors, sigma $=100$, (diffusion map) sigma $=300$, and alpha $=1$. These were set in such a way that they reveal a similar structure as the kernel map with reference point method.

\section{CONCLUSION}

In recent years, considerable progress has been made in the area of data visualization and dimensionality reduction using kernel eigenmap methods. In this paper, we have shown how to characterize solutions by linear systems instead of eigenvalue problems. For this purpose, we proposed a new method of kernel maps with a reference point. The framework of LS-SVMs is adopted, which naturally allows to incorporate reference point constraints in the primal problem within the constrained optimization problem. Kernel model representations are generated in a systematic and straightforward way in the dual. The optimal coordinates for visualization in the low-dimensional space are computed from a linear system in a numerically reliable way. The framework immediately allows for out-of-sample extensions. Validation-based learning of the tuning parameters has been demonstrated with good results on toy problems and real-life data sets.

\section{APPENDIX}

\section{Proof of Lemma 1}

The solution (13) is obtained in a straightforward way. First, construct the Lagrangian

$$
\begin{aligned}
\mathcal{L}\left(z, w_{1}, w_{2}, e_{i, 1}, e_{i, 2} ; \alpha_{i, 1}, \alpha_{i, 2}\right) & \\
= & J_{3}+\sum_{i=1}^{N} \alpha_{i, 1}\left(c_{i, 1}^{T} z-w_{1}^{T} \varphi_{1}\left(x_{i}\right)-e_{i, 1}\right) \\
& +\sum_{i=1}^{N} \alpha_{i, 2}\left(c_{i, 2}^{T} z-w_{2}^{T} \varphi_{2}\left(x_{i}\right)-e_{i, 2}\right) .
\end{aligned}
$$

For characterization of the local minima, take the conditions for optimality $\partial \mathcal{L} / \partial z=0, \partial \mathcal{L} / \partial w_{1}=0, \partial \mathcal{L} / \partial w_{2}=0$, 


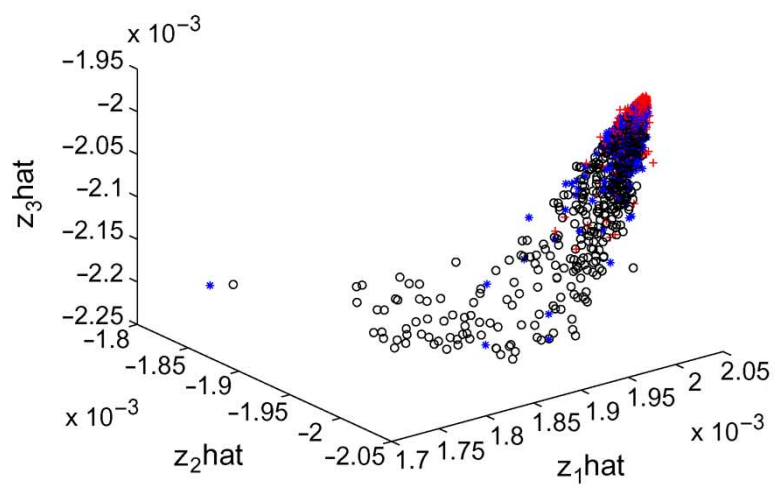

(a)

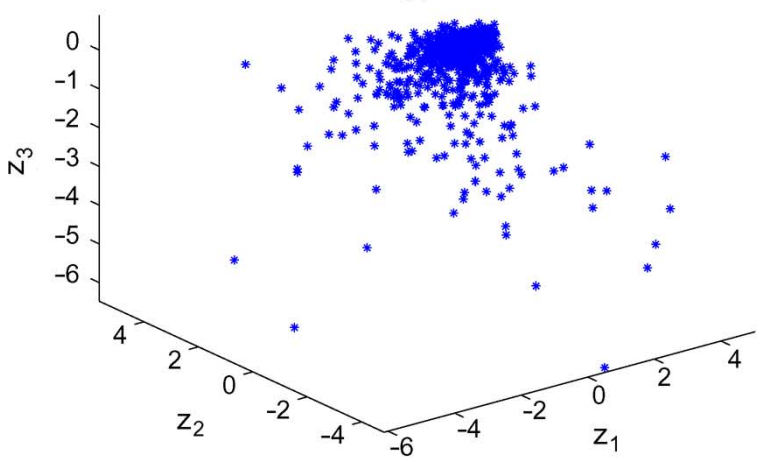

(c)

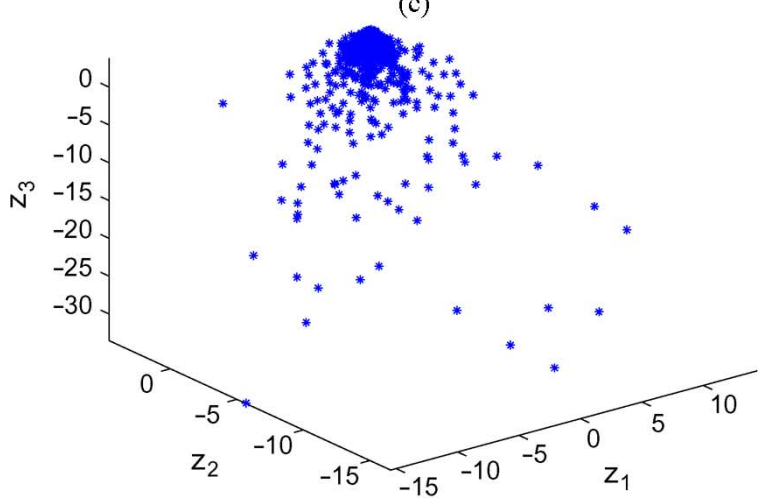

(e)

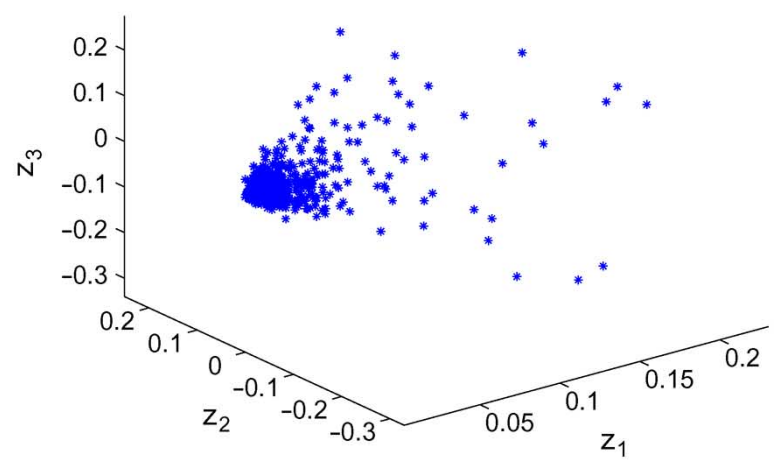

(b)

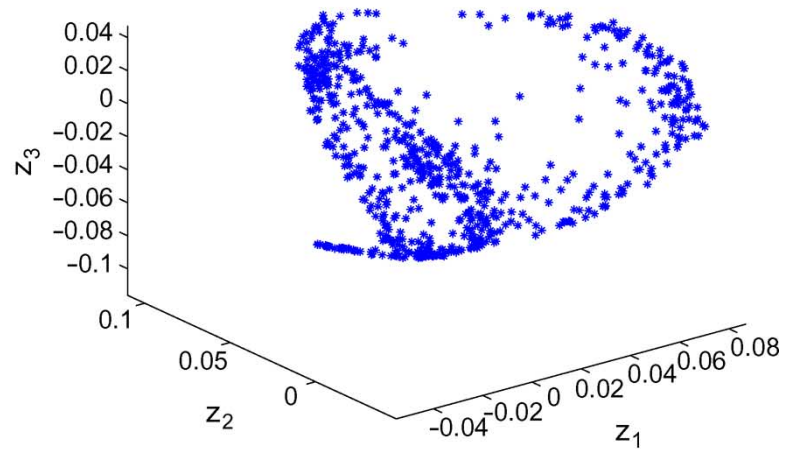

(d)

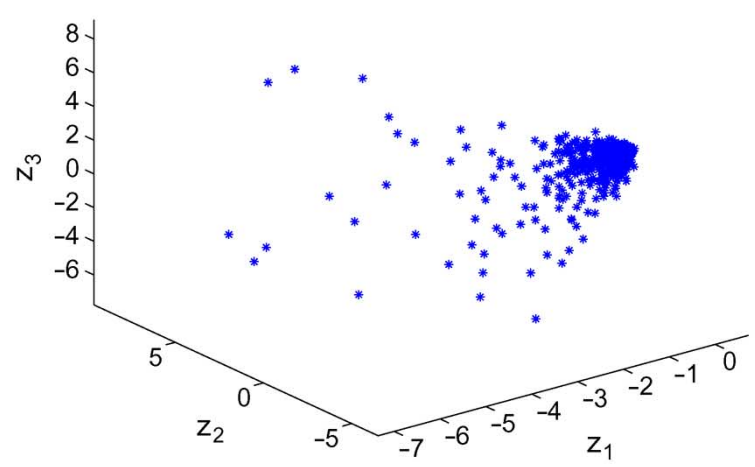

(f)

Fig. 8. Alon data set: comparison of different methods. (a) Kernel map with reference point $[-1 ;+1 ;+1]$, (b) PCA, (c) LLE, (d) Laplacian eigenmap, (e) MDS, and (f) diffusion map. Tuning parameters were set to reveal similar structure as the kernel map with reference point method.

$\partial \mathcal{L} / \partial e_{i, 1}=0, \partial \mathcal{L} / \partial e_{i, 2}=0, \partial \mathcal{L} / \partial \alpha_{i, 1}=0$, and $\partial \mathcal{L} / \partial \alpha_{i, 2}=$ 0 . This leads to the following set of equations, which should be satisfied simultaneously:

$$
\left\{\begin{array}{l}
\frac{\partial \mathcal{L}}{\partial z}=-\gamma z+R z+\sum_{i=1}^{N} \alpha_{i, 1} c_{i, 1}+\sum_{i=1}^{N} \alpha_{i, 2} c_{i, 2}=0 \\
\frac{\partial \mathcal{L}}{\partial w_{1}}=\nu w_{1}-\sum_{i=1}^{N} \alpha_{i, 1} \varphi_{1}\left(x_{i}\right)=0 \\
\frac{\partial \mathcal{L}}{\partial w_{2}}=\nu w_{2}-\sum_{i=1}^{N} \alpha_{i, 2} \varphi_{2}\left(x_{i}\right)=0 \\
\frac{\partial \mathcal{L}}{\partial e_{i, 1}}=\eta e_{i, 1}-\alpha_{i, 1}=0, i=1, \ldots, N \\
\frac{\partial \mathcal{L}}{\partial e_{i, 2}}=\eta e_{i, 2}-\alpha_{i, 2}=0, i=1, \ldots, N \\
\frac{\partial \mathcal{L}}{\partial \alpha_{i, 1}}=c_{i, 1}^{T} z-w_{1}^{T} \varphi_{1}\left(x_{i}\right)-e_{i, 1}, i=1, \ldots, N \\
\frac{\partial \mathcal{L}}{\partial \alpha_{i, 2}}=c_{i, 2}^{T} z-w_{2}^{T} \varphi_{2}\left(x_{i}\right)-e_{i, 2}, i=1, \ldots, N .
\end{array}\right.
$$

After elimination of $w_{1}, w_{2}, e_{i, 1}, e_{i, 2}$ and application of the kernel trick, the set of equations can be expressed in terms of $z, \alpha_{1}, \alpha_{2}$. One obtains

$$
-\gamma z+R z+V_{1} \alpha_{1}+V_{2} \alpha_{2}=0
$$

and

$$
\begin{aligned}
& V_{1}^{T} z-\frac{1}{\nu} \Omega_{1} \alpha_{1}-\frac{1}{\eta} \alpha_{1}=0 \\
& V_{2}^{T} z-\frac{1}{\nu} \Omega_{2} \alpha_{2}-\frac{1}{\eta} \alpha_{2}=0 .
\end{aligned}
$$

The dual model representation follows from the conditions for optimality. 

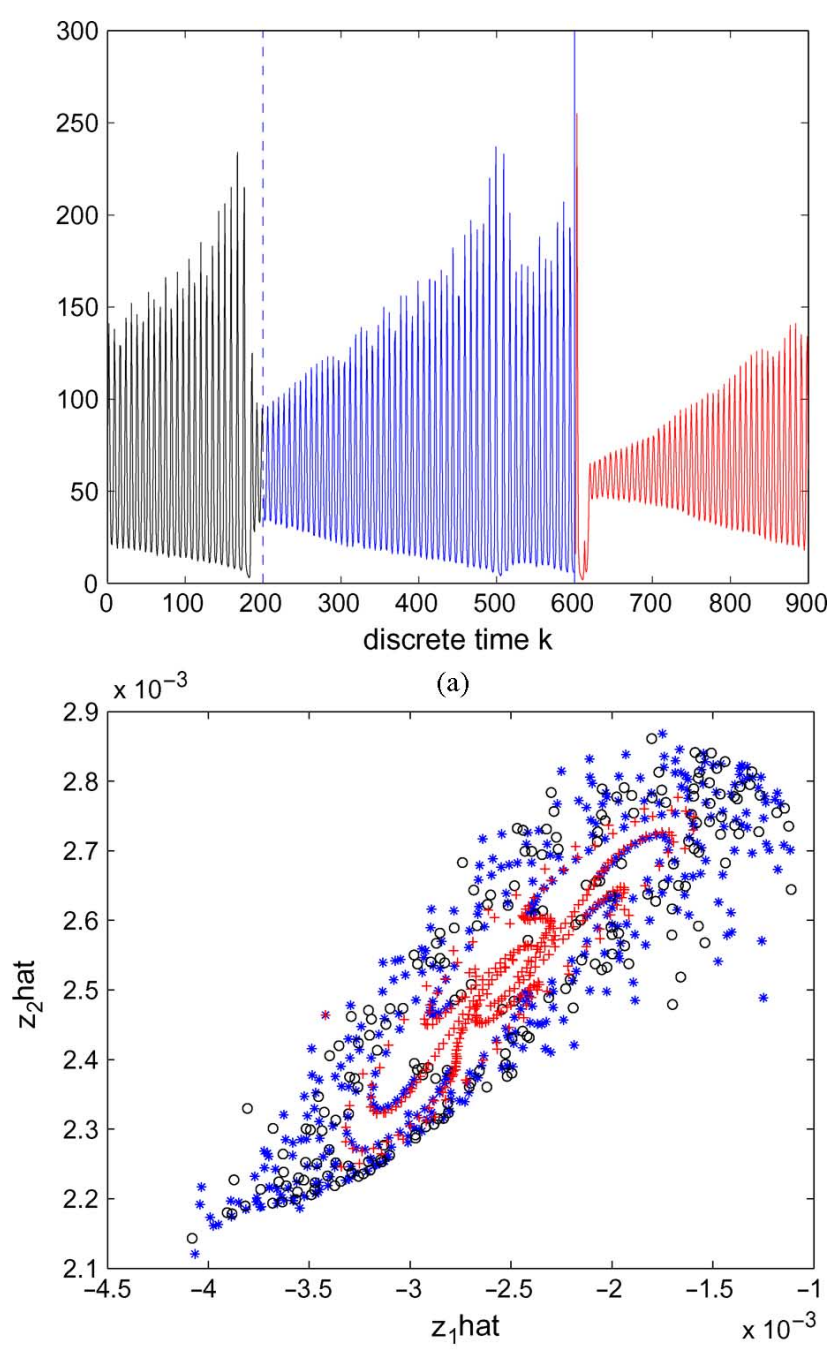

(b)

Fig. 9. (a) Santa Fe chaotic laser data in time: validation set (black)—-training set (blue) - test set (red); (b) 2-D visualization obtained by the kernel map reference point method [training data (blue $*$ ), validation data (black o), and test data $($ red +$)]$.

\section{Proof of Lemma 2}

The solution (18) is obtained in a straightforward way. Construct the Lagrangian

$$
\begin{aligned}
\mathcal{L}\left(z, w_{1}, w_{2}, b_{1}, b_{2}, e_{i, 1}, e_{i, 2} ; \beta_{1,1}, \beta_{1,2}, \alpha_{i, 1}, \alpha_{i, 2}\right) & \\
= & J_{4}+\beta_{1,1}\left(c_{1,1}^{T} z-q_{1}-e_{1,1}\right)+\beta_{1,2}\left(c_{1,2}^{T} z-q_{2}-e_{1,2}\right) \\
& +\sum_{i=2}^{N} \alpha_{i, 1}\left(c_{i, 1}^{T} z-w_{1}^{T} \varphi_{1}\left(x_{i}\right)-b_{1}-e_{i, 1}\right) \\
& +\sum_{i=2}^{N} \alpha_{i, 2}\left(c_{i, 2}^{T} z-w_{2}^{T} \varphi_{2}\left(x_{i}\right)-b_{2}-e_{i, 2}\right) .
\end{aligned}
$$

Taking the conditions for optimality [11] $\partial \mathcal{L} / \partial z=0$, $\partial \mathcal{L} / \partial w_{1}=0, \partial \mathcal{L} / \partial w_{2}=0, \partial \mathcal{L} / \partial b_{1}=0, \partial \mathcal{L} / \partial b_{2}=0$, $\partial \mathcal{L} / \partial e_{i, 1}=0, \partial \mathcal{L} / \partial e_{i, 2}=0, \partial \mathcal{L} / \partial \beta_{1,1}=0, \partial \mathcal{L} / \partial \beta_{1,2}=0$, $\partial \mathcal{L} / \partial \alpha_{i, 1}=0$, and $\partial \mathcal{L} / \partial \alpha_{i, 2}=0$, this gives the following set of equations, which should be satisfied simultaneously:

$$
\left\{\begin{aligned}
& \frac{\partial \mathcal{L}}{\partial z}=-\gamma z+\left(I-P_{D}\right)^{T}\left(I-P_{D}\right) z+\beta_{1,1} c_{1,1}+\beta_{1,2} c_{1,2} \\
&+\sum_{i=2}^{N} \alpha_{i, 1} c_{i, 1}+\sum_{i=2}^{N} \alpha_{i, 2} c_{i, 2}=0 \\
& \frac{\partial \mathcal{L}}{\partial w_{1}}=\nu w_{1}-\sum_{i=2}^{N} \alpha_{i, 1} \varphi_{1}\left(x_{i}\right)=0 \frac{\partial \mathcal{L}}{\partial w_{2}}=\nu w_{2}-\sum_{i=2}^{N} \alpha_{i, 2} \varphi_{2}\left(x_{i}\right)=0 \\
& \frac{\partial \mathcal{L}}{\partial b_{1}}=\sum_{i=2}^{N} \alpha_{i, 1}=1_{N-1}^{T} \alpha_{1}=0 \\
& \frac{\partial \mathcal{L}}{\partial b_{2}}=\sum_{i=2}^{N} \alpha_{i, 2}=1_{N-1}^{T} \alpha_{2}=0 \\
& \frac{\partial \mathcal{L}}{\partial e_{1,1}}=\eta e_{1,1}-\beta_{1,1}=0 \\
& \frac{\partial \mathcal{L}}{\partial e_{1,2}}=\eta e_{1,2}-\beta_{1,2}=0 \\
& \frac{\partial \mathcal{L}}{\partial e_{i, 1}}=\eta e_{i, 1}-\alpha_{i, 1}=0, i=2, \ldots, N \\
& \frac{\partial \mathcal{L}}{\partial e_{i, 2}}=\eta e_{i, 2}-\alpha_{i, 2}=0, i=2, \ldots, N \\
& \frac{\partial \mathcal{L}}{\partial \beta_{1,1}}=c_{1,1}^{T} z-q_{1}-e_{1,1}=0 \\
& \frac{\partial \mathcal{L}}{\partial \beta_{1,2}}=c_{1,2}^{T} z-q_{2}-e_{1,2}=0 \\
& \frac{\partial \mathcal{L}}{\partial \alpha_{i, 1}}=c_{i, 1}^{T} z-w_{1}^{T} \varphi_{1}\left(x_{i}\right)-b_{1}-e_{i, 1}=0, i=2, \ldots, N \\
& \frac{\partial \mathcal{L}}{\partial \alpha_{i, 2}}=c_{i, 2}^{T} z-w_{2}^{T} \varphi_{2}\left(x_{i}\right)-b_{2}-e_{i, 2}=0, i=2, \ldots, N .
\end{aligned}\right.
$$

After elimination of $w_{1}, w_{2}, e_{i, 1}, e_{i, 2}$ and application of the kernel trick, the set of equations can be expressed in terms of $z, b_{1}, b_{2}, \alpha_{1}, \alpha_{2}$. One obtains

$$
\begin{array}{r}
-\gamma z+\left(I-P_{D}\right)^{T}\left(I-P_{D}\right) z+V_{1} \alpha_{1}+V_{2} \alpha_{2}+\eta c_{1,1} c_{1,1}^{T} z+ \\
\eta c_{1,2} c_{1,2}^{T} z=\eta\left(q_{1} c_{1,1}+q_{2} c_{1,2}\right)
\end{array}
$$

and

$$
\begin{aligned}
V_{1}^{T} z-\frac{1}{\nu} \Omega_{1} \alpha_{1}-\frac{1}{\eta} \alpha_{1}-b_{1} 1_{N-1} & =0 \\
V_{2}^{T} z-\frac{1}{\nu} \Omega_{2} \alpha_{2}-\frac{1}{\eta} \alpha_{2}-b_{2} 1_{N-1} & =0 \\
\beta_{1,1} & =\eta\left(c_{1,1}^{T} z-q_{1}\right) \\
\beta_{1,2} & =\eta\left(c_{1,2}^{T} z-q_{2}\right) .
\end{aligned}
$$

The dual model representation follows from the conditions for optimality.

\section{Proof of Lemma 3}

The proof is similar to the proof of Lemma 2. The Lagrangian is given by

$$
\begin{array}{r}
\mathcal{L}\left(z, w_{j}, b_{j}, e_{i, j} ; \beta_{1, j}, \alpha_{i, j}\right)=J_{5}+\sum_{j=1}^{d} \beta_{1, j}\left(c_{1, j}^{T} z-q_{j}-e_{1, j}\right) \\
+\sum_{j=1}^{d} \sum_{i=2}^{N} \alpha_{i, j}\left(c_{i, j}^{T} z-w_{j}^{T} \varphi_{j}\left(x_{i}\right)-b_{j}-e_{i, j}\right) .
\end{array}
$$




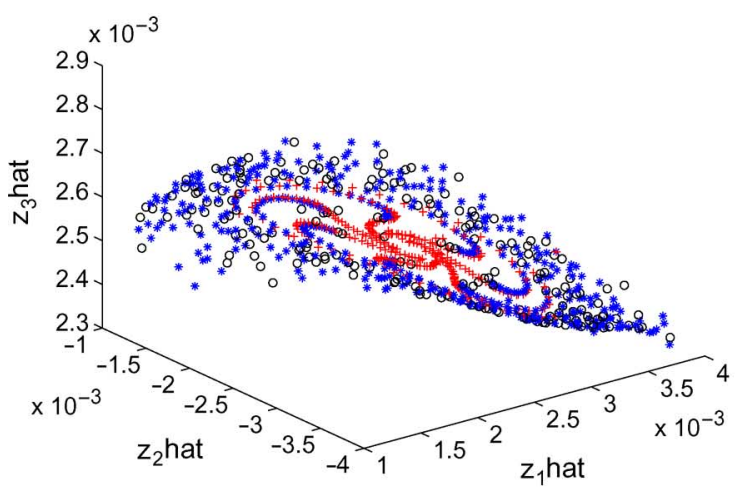

(a)

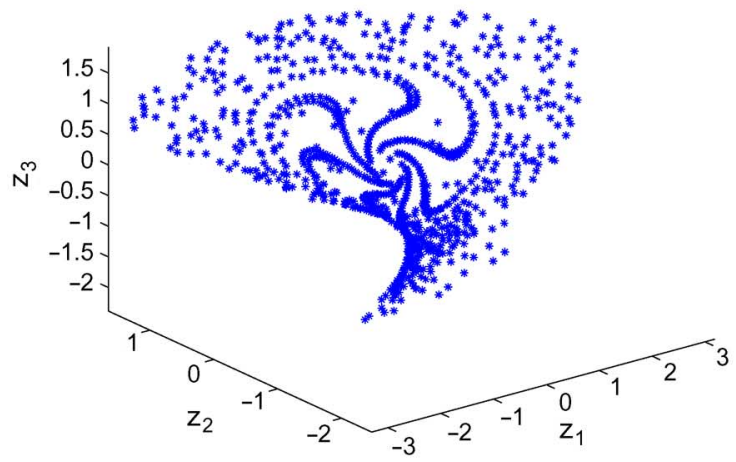

(c)

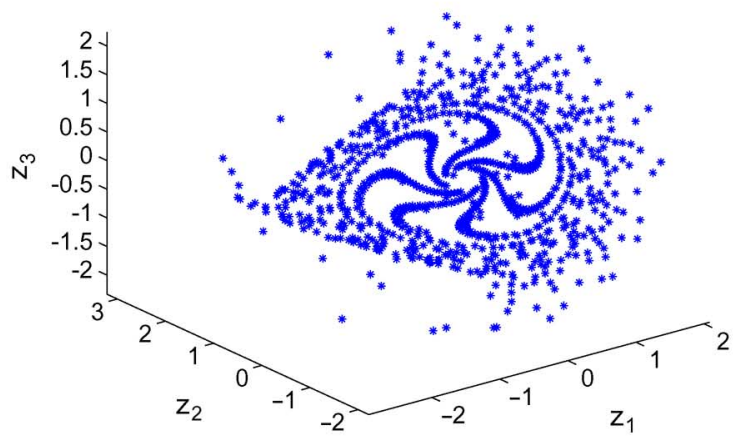

(e)

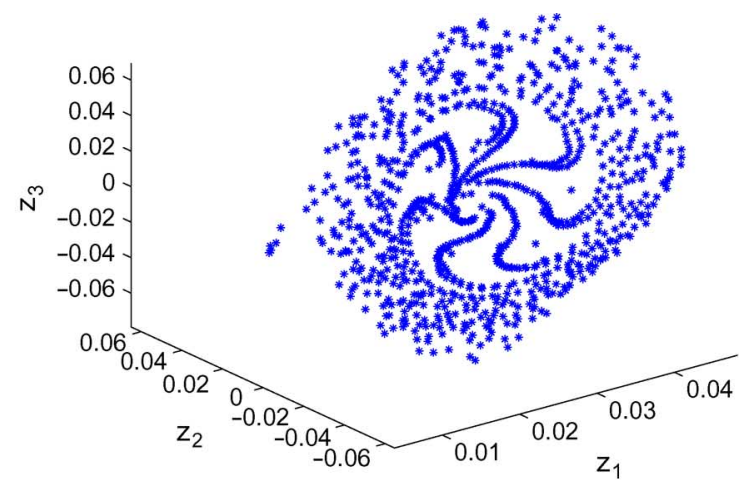

(b)

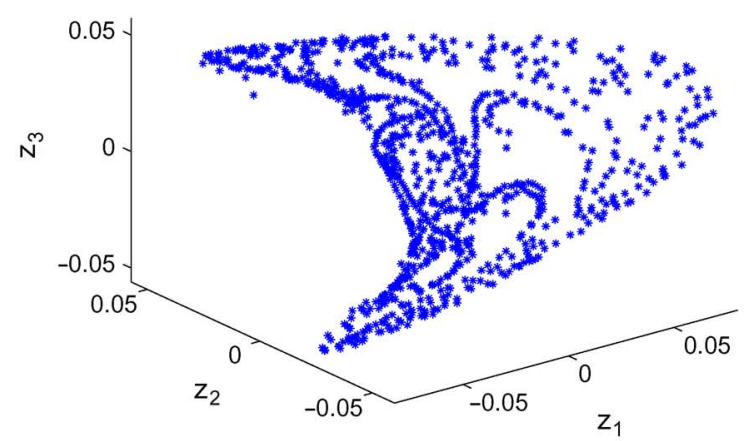

(d)

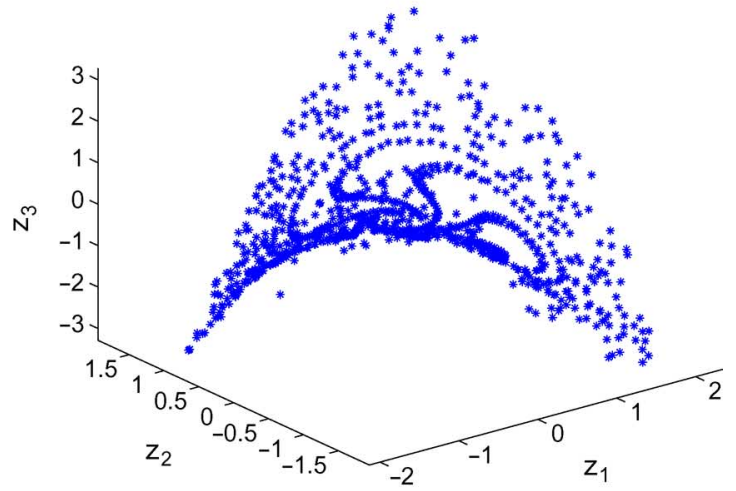

(f)

Fig. 10. Santa Fe data set: comparison with existing methods. (a) Kernel map with reference point $[-1 ;+1 ;-1]$, (b) PCA, (c) LLE with 100 nearest neighbors, (d) Laplacian eigenmap with 300 nearest neighbors (note the large number needed in this example), (e) MDS, and (f) diffusion map. Tuning parameters were set to reveal similar structure as the kernel map with reference point method.

From the conditions for optimality $\partial \mathcal{L} / \partial z=0, \partial \mathcal{L} / \partial w_{j}=0$, $\partial \mathcal{L} / \partial b_{j}=0, \partial \mathcal{L} / \partial e_{i, j}=0, \partial \mathcal{L} / \partial \beta_{1, j}=0$, and $\partial \mathcal{L} / \partial \alpha_{i, j}=$ 0 , one obtains

$$
\left\{\begin{aligned}
& \frac{\partial \mathcal{L}}{\partial z}=-\gamma z+\left(I-P_{D}\right)^{T}\left(I-P_{D}\right) z+\sum_{j=1}^{d} \beta_{1, j} c_{1, j} \\
&+\sum_{j=1}^{d} \sum_{i=2}^{N} \alpha_{i, j} c_{i, j}=0 \\
& \frac{\partial \mathcal{L}}{\partial w_{j}}=\nu w_{j}-\sum_{i=2}^{N} \alpha_{i, j} \varphi_{j}\left(x_{i}\right)=0, j=1, \ldots, d \\
& \frac{\partial \mathcal{L}}{\partial b_{j}}=\sum_{i=2}^{N} \alpha_{i, j}=1_{N-1}^{T} \alpha_{j}=0, j=1, \ldots, d \\
& \frac{\partial \mathcal{L}}{\partial e_{1, j}}=\eta e_{1, j}-\beta_{1, j}=0, j=1, \ldots, d \\
& \frac{\partial \mathcal{L}}{\partial e_{i, j}}=\eta e_{i, j}-\alpha_{i, j}=0, i=2, \ldots, N, j=1, \ldots, d \\
& \frac{\partial \mathcal{L}}{\partial \beta_{1, j}}=c_{1, j}^{T} z-q_{j}-e_{1, j}=0, j=1, \ldots, d \\
& \frac{\partial \mathcal{L}}{\partial \alpha_{i, j}}=c_{i, j}^{T} z-w_{j}^{T} \varphi_{j}\left(x_{i}\right)-b_{j}-e_{i, j}=0, \\
& i=2, \ldots, N, j=1, \ldots, d .
\end{aligned}\right.
$$

After elimination of $w_{j}, e_{i, j}$ and application of the kernel trick, the set of equations can be expressed in terms of $z, b_{j}, \alpha_{j}$. This gives

$$
\begin{aligned}
-\gamma z+\left(I-P_{D}\right)^{T}\left(I-P_{D}\right) z & +\sum_{j=1}^{d} V_{j} \alpha_{j} \\
& +\eta \sum_{j=1}^{d} c_{1, j} c_{1, j}^{T} z=\eta \sum_{j=1}^{d} q_{j} c_{1, j}
\end{aligned}
$$

and 
The dual model representation follows from the conditions for optimality.

\section{REFERENCES}

[1] C. Alzate and J. A. K. Suykens, "A weighted kernel PCA formulation with out-of-sample extensions for spectral clustering methods," in Proc. Int. Joint Conf. Neural Netw., Vancouver, BC, Canada, 2006, pp. $138-144$.

[2] M. Belkin and P. Niyogi, "Laplacian eigenmaps for dimensionality reduction and data representation," Neural Comput., vol. 15, no. 6, pp. 1373-1396, Jun. 2003.

[3] M. Belkin, P. Niyogi, and V. Sindhwani, "Manifold regularization: A geometric framework for learning from labeled and unlabeled examples," J. Mach. Learn. Res., vol. 7, pp. 2399-2434, 2006.

[4] Y. Bengio, J.-F. Paiement, P. Vincent, O. Delalleau, N. Le Roux, and M. Ouimet, "Out-of-sample extensions for LLE, isomap, MDS, eigenmaps, and spectral clustering," in Advances in Neural Information Processing Systems. Cambridge, MA: MIT Press, 2004, vol. 16.

[5] G. C. Cawley and N. L. C. Talbot, "Fast exact leave-one-out cross-validation of sparse least-squares support vector machines," Neural Netw., vol. 17, no. 10, pp. 1467-1475, 2004.

[6] R. R. Coifman, S. Lafon, A. B. Lee, M. Maggioni, B. Nadler, F. Warner, and S. W. Zucker, "Geometric diffusions as a tool for harmonic analysis and structure definition of data: Diffusion maps," Proc. Nat. Acad. Sci. USA, vol. 102, no. 21, pp. 7426-7431, May 2005.

[7] R. R. Coifman and S. Lafon, "Diffusion maps," Appl. Comput. Harmonic Anal., vol. 21, no. 1, pp. 5-30, 2006.

[8] F. Cucker and S. Smale, "On the mathematical foundations of learning theory," Bull. AMS, vol. 39, pp. 1-49, 2002.

[9] D. L. Donoho and C. Grimes, "Hessian eigenmaps: Locally linear embedding techniques for high-dimensional data," Proc. Nat. Acad. Sci. USA, vol. 100, no. 10, pp. 5591-5596, May 2003.

[10] T. Evgeniou, M. Pontil, and T. Poggio, "Regularization networks and support vector machines," Adv. Comput. Math., vol. 13, no. 1, pp. 1-50, 2000.

[11] R. Fletcher, Practical Methods of Optimization. New York: Wiley, 1987.

[12] G. H. Golub and C. F. Van Loan, Matrix Computations. Baltimore, MD: The Johns Hopkins Univ. Press, 1989.

[13] I. T. Jolliffe, Principal Component Analysis, ser. Statistics. New York: Springer-Verlag, 1986.

[14] G. E. Hinton and R. R. Salakhutdinov, "Reducing the dimensionality of data with neural networks," Science, vol. 313, no. 5786, pp. 504-507, Jul. 2006.

[15] T. Kohonen, "The self-organizing map," Proc. IEEE, vol. 78, no. 9, pp. 1464-1480, Sep. 1990.

[16] S. Roweis and L. Saul, "Nonlinear dimensionality reduction by locally linear embedding," Science, vol. 290, no. 5500, pp. 2323-2326, Dec. 2000.

[17] L. Saul, K. Q. Weinberger, F. Sha, J. Ham, and D. D. Lee, "Spectral methods for dimensionality reduction," in Semi-Supervised Learning, O. Chapelle, B. Schölkopf, and A. Zien, Eds. Cambridge MA: MIT Press, 2006, ch. 16, pp. 293-308.

[18] C. Saunders, A. Gammerman, and V. Vovk, "Ridge regression learning algorithm in dual variables," in Proc. 15th Int. Conf. Mach. Learn., Madison, WI, 1998, pp. 515-521.

[19] B. Schölkopf, A. Smola, and K.-R. Müller, "Nonlinear component analysis as a kernel eigenvalue problem," Neural Comput., vol. 10, pp. 1299-1319, 1998.

[20] B. Schölkopf and A. Smola, Learning With Kernels. Cambridge, MA: MIT Press, 2002.
[21] J. Shawe-Taylor and N. Cristianini, Kernel Methods for Pattern Analysis. Cambridge, U.K.: Cambridge Univ. Press, Jun. 2004.

[22] S. Smale, "Complexity theory and numerical analysis," Acta Numerica, pp. 523-551, 1997.

[23] J. A. K. Suykens and J. Vandewalle, "Least squares support vector machine classifiers," Neural Process. Lett., vol. 9, no. 3, pp. 293-300, 1999.

[24] J. A. K. Suykens, T. Van Gestel, J. Vandewalle, and B. De Moor, "A support vector machine formulation to PCA analysis and its kernel version," IEEE Trans. Neural Netw., vol. 14, no. 2, pp. 447-450, Mar. 2003.

[25] J. A. K. Suykens, J. Vandewalle, and B. De Moor, "Optimal control by least squares support vector machines," Neural Netw., vol. 14, no. 1 , pp. 23-35, 2001.

[26] J. A. K. Suykens, T. Van Gestel, J. De Brabanter, B. De Moor, and J. Vandewalle, Least Squares Support Vector Machines. , Singapore: World Scientific, 2002.

[27] J. B. Tenenbaum, V. De Silva, and J. C. Langford, "A global geometric framework for nonlinear dimensionality reduction," Science, vol. 290, no. 5500 , pp. 2319-2323, 2000.

[28] V. Vapnik, Statistical Learning Theory. New York: Wiley, 1998.

[29] J. P. Vert and Y. Yamanishi, "Supervised graph inference," in Advances in Neural Information Processing Systems. Cambridge, MA: MIT Press, 2005, vol. 17, pp. 1433-1440.

[30] G. Wahba, Spline Models for Observational Data, ser. Applied Mathematics. Philadelphia, PA: SIAM, 1990, vol. 59.

[31] K. Q. Weinberger and L. K. Saul, "Unsupervised learning of image manifolds by semidefinite programming," in Proc. IEEE Conf. Comput. Vis. Pattern Recognit., Washington, DC, 2004, vol. 2, pp. 988-995.

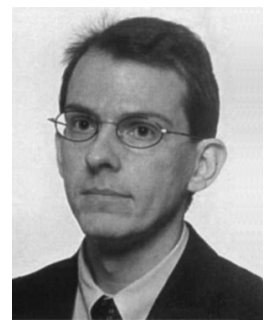

Johan A. K. Suykens (SM'05) was born in Willebroek, Belgium, on May 18, 1966. He received the degree in electro-mechanical engineering and the Ph.D. degree in applied sciences from the Katholieke Universiteit Leuven, Leuven, Belgium, in 1989 and 1995 , respectively.

In 1996, he was a Visiting Postdoctoral Researcher at the University of California, Berkeley. He has been a Postdoctoral Researcher with the Fund for Scientific Research FWO Flanders and currently is a Professor (Hoogleraar) at the Katholieke Universiteit Leuven. He is author of the books Artificial Neural Networks for Modelling and Control of Non-linear Systems (Norwell, MA: Kluwer, 1996) and Least Squares Support Vector Machines (Singapore: World Scientific, 2002), coauthor of the book Cellular Neural Networks, Multi-Scroll Chaos and Synchronization (Singapore: World Scientific, 2005) and editor of the books Nonlinear Modeling: Advanced Black-Box Techniques (Norwell, MA: Kluwer, 1998) and Advances in Learning Theory: Methods, Models and Applications (Amsterdam, The Netherlands: IOS Press, 2003). His research interests are mainly in the areas of the theory and application of neural networks and nonlinear systems.

Dr. Suykens has served as an Associate Editor for the IEEE TRANSACTIONS ON CIRCUITS AND SYSTEMS (1997-1999 and 2004-2007) and since 1998 he has been serving as an Associate Editor for the IEEE TRANSACTIONS ON NEURAL NETWORKS. In 1998, he organized an International Workshop on Nonlinear Modelling with Time-Series Prediction Competition. He received an IEEE Signal Processing Society 1999 Best Paper (Senior) Award and several Best Paper Awards at international conferences. He is a recipient of the International Neural Networks Society (INNS) 2000 Young Investigator Award for significant contributions in the field of neural networks. He has served as a Director and Organizer of the NATO Advanced Study Institute on Learning Theory and Practice (Leuven, Belgium, 2002), as a Program Cochair for the International Joint Conference on Neural Networks 2004 and the International Symposium on Nonlinear Theory and its Applications 2005, and as an Organizer of the International Symposium on Synchronization in Complex Networks 2007. 